\title{
P. Edulis Extract Protects Against Amyloid- $\beta$ Toxicity in Alzheimer's Disease Models Through Maintenance of Mitochondrial Homeostasis via the FOX03/DAF-16 Pathway
}

\section{Shu-qin Cao}

Chulalongkorn University Faculty of Allied Health Sciences

\section{Yahyah Aman}

University of Oslo Faculty of Medicine: Universitetet i Oslo Det medisinske fakultet

\section{Evandro Fei Fang}

University of Oslo Faculty of Medicine: Universitetet i Oslo Det medisinske fakultet

Tewin Tencomnao ( $\nabla$ tewin.t@chula.ac.th )

Chulalongkorn University Faculty of Allied Health Sciences https://orcid.org/0000-0003-4177-2165

\section{Research Article}

Keywords: Alzheimer's disease, Glutamatergic neurons, DAF-16, mitophagy, DCT-1

Posted Date: December 30th, 2021

DOI: https://doi.org/10.21203/rs.3.rs-1208060/v1

License: (c) (i) This work is licensed under a Creative Commons Attribution 4.0 International License.

Read Full License 


\section{Abstract}

Alzheimer's disease (AD) is a common and devastating disease characterized by pathological aggregations of beta-amyloid $(A \beta)$ plaques extracellularly, and Tau tangles intracellularly. While our understandings of the aetiologies of $A D$ have greatly expanded over the decades, there is no drug available to stop disease progression. Here, we demonstrate the potential of $P$. edulis pericarp extract in protecting against $\mathrm{A} \beta$-mediated neurotoxicity in mammalian cells and Caenorhabditis elegans models of AD. We show $P$. edulis pericarp protects against memory deficit, neuronal loss, and promotes longevity in the $A \beta$ model of $A D$ via stimulation of mitophagy, a selective cellular clearance of damaged and dysfunctional mitochondria. P. edulis pericarp also restores memory and increases neuronal resilience in a $C$. elegans Tau model of AD. While defective mitophagy-induced accumulation of damaged mitochondria contributes to AD progression, $P$. edulis pericarp improves mitochondrial homeostasis through NIX/DCT1-dependent mitophagy and SOD3-dependent mitochondrial resilience, both via increased nuclear translocation of the upstream transcriptional regulator FOXO3/DAF-16. Further studies to identify active molecules in $P$. edulis pericarp that could maintain neuronal mitochondrial homeostasis may enable the development of potential drug candidates for AD.

\section{Introduction}

Alzheimer's disease (AD) is a progressive and irreversible disease of the central nervous system (CNS). It is the most common form of dementia which affecting around 50 million people globally at present, a figure estimated to triple by 2050 [1]. Clinically it is characterized by an insidious onset and progressive deterioration of cognitive function [1,2]. The pathological hallmarks of the disease include formation of extracellular plaques composed of aggregated beta-amyloid $(A \beta)$ and accumulation of intracellular tau in the form of neurofibrillary tangles [3-7]. These pathological features are accompanied by neuroinflammation, mitochondrial dysfunction, synaptic degeneration, and neuronal loss due to necroptosis [8-13]. To date, cholinesterase inhibitors and glutamate receptor antagonists have been the standard drugs for the treatment of $A D$. These therapeutic interventions provide symptomatic relief, but are incapable of curing and/or delaying the progression of the disease. Therefore, there is a dire need for identification of novel therapeutic strategies to counter AD.

Passiflora edulis (P. edulis), commonly known as passion fruit, is natively to the Southern American, but widely cultivated in tropical and subtropical areas worldwide. The pulp and pericarp of the passion fruit are a source of phytochemical contents such as polyphenols, triterpenoids, glycosides, carotenoids, polysaccharides, aromatic oils, and essential nutrients [14-18]. Pharmacological studies have identified the bioactivities of passion fruit including anti-oxidative, anti-inflammatory, anti-diabetic, and potentially hepatoprotective effects [19-23]. Additionally, it has been reported that passion fruit extracts act as a modulator of the glutamatergic system, which further promote neuroprotective activities has been reported $[24,25]$. However, the underlying mechanism of the neurotherapeutic activity of $P$. edulis extract has remained elusive. In this study, we wanted to determine whether administration of $P$. edulis extract could inhibit memory loss and pathological phenotypes in Caenorhabditis elegans ( $C$. elegans) models of 
AD. We further evaluated the underling molecular mechanisms in both $C$. elegans and mammalian cell systems.

\section{Results}

P. edulis pericarp extract attenuates memory loss and prolongs lifespan in AD C. elegans

Progressive memory impairment is the most common symptom in AD patients [26]. Thus, we set out to evaluate whether the $P$. edulis pericarp (PEP) extract can inhibit memory loss in the transgenic $C$. elegans models of $A D$ harboring pan neuronal human $A \beta_{1-42}\left(J K M 2, h A \beta_{1-42}\right)$ or pan-neuronal expression of human P301L Tau mutation (CK12, hTau[P301L]). For this purpose, we utilized an aversive olfactory learning chemotaxis assay (a negative value correlates with positive chemotaxis-related memory). Transgenic nematodes expressing $\mathrm{hA} \beta_{1-42}$ and $\mathrm{hTau}[\mathrm{P} 301 \mathrm{~L}]$ displayed severe cognitive deficits and neurodegeneration as we $[10,27]$ and others $[28,29]$ reported before. We administrated PEP at $250 \mu \mathrm{g} / \mathrm{ml}$ to the nematodes from egg hatching onwards and performed memory experiments on adult Day 1 . While the $h A \beta_{1-42}$ and $h T a u[P 301 L]$ animals had impaired memory, PEP inhibited memory loss in these AD nematodes; to note, PEP did not influence the memory of WT (N2) animals (Fig. 1a). Epidemiological studies indicate that AD not only impairs memory but also shortens lifespan [30,31]. We postulated that strategies that improve memory in animals with AD could also extend their lifespan [27]. Therefore, we subsequently assess the potential effect of PEP on lifespan in the transgenic nematode models of AD. As expected, in the transgenic $C$. elegans models of $A D$, both $h A \beta_{1-42}$ and $h T a u[P 301 L]$ models exhibited a shorter lifespan in comparison to WT control (Fig. 1b). Upon PEP administration, only hA $\beta_{1-42}$ nematodes displayed a significant extension of lifespan, with no influence of PEP extract on the lifespan of hTau[P301L] and the WT animals (Fig. 1c-e). A summary of the lifespan data in different groups was in Supplementary Table 1. These findings indicate PEP protected against memory deficits and extended lifespan in particular the $h A \beta_{1-42}$ model of $A D$.

\section{P. edulis extract inhibits neurodegeneration in AD C. elegans and cells}

Having established the potential of PEP extract to improve healthspan and lifespan in the $C$. elegans $h A \beta_{1-42}$ model of $A D$, we set out to investigate the underlying mechanism. For this purpose, we first evaluated whether PEP potentiates neuroprotection that results in the improved functional behavior. The two major neurotransmission systems primarily affected in $A D$ are the cholinergic and the glutamatergic systems [32-34]. Cholinergic neurons play a key role in the CNS, and acetylcholine (ACh) works as neurotransmitter that serviced all cholinergic neurons. There is a likelihood that either Ach depletion or hyper-accumulation links to neurodegeneration [35-37]. The functional activity of the cholinergic system in the AD nematodes was assessed by feeding the animals with aldicarb, an acetylcholinesterase inhibitor that induces hyper-accumulation of Ach, resulting in accelerated skeletal muscle contraction, and finally paralysis [38]. Controls for the assay, in the form of aldicarb hypersensitive (VC233: tom1(ok285)I) and aldicarb resistant (NM204: snt-1(md290)II) strains displayed increased and reduced sensitivities to aldicarb, respectively, compared to the WT nematodes (Fig. 2a). The hA $\beta_{1-42}$ model of AD 
displayed an increased sensitivity to aldicarb compared to the WT N2; whilst hTau[P301L] nematodes did not show increased sensitivity to aldicarb compared to that of WT animals (Fig. 2a). These findings suggest an impairment in the cholinergic system in $h A \beta_{1-42}$ nematodes. Application of PEP resulted in a delay in aldicarb-mediated paralysis in both $h A \beta_{1-42}$ and $h T a u[p 3011]$ models of $A D$, as well as, in the WT $\mathrm{N} 2$ nematodes (Fig. 2b-d). This implies that PEP enhanced cholinergic neuronal resistance to aldicarb in both pathological and physiological conditions.

In addition to cholinergic neuronal protection, we asked whether PEP could protect against degeneration of the glutamatergic neurons in AD. Glutamatergic neurons are another vital type of neurons found in the $C N S$, and are impaired in $A D[39,40]$. $A \beta$ induces glutamatergic neuronal loss and promotes $A D$ progresses $[40,41]$. To evaluate whether PEP could protect against $A \beta$-induced neurodegeneration in the glutamatergic sub-type neurons, we used a series of well-characterized nematode models whereby $h A \beta_{1-42}$ is only expressed in the glutamatergic neurons and induces neurodegeneration [27, 42]. Five taillocalized glutamatergic neurons [LUA(R), LUA(L), PVR, PLM(R), and PLM(L)] ewere used for data quantification as these five neurons show clear, stable and easy-to-quantify patterns of neurodegeneration [42]. As reported before [27, 42], transgenic nematodes carrying hA $\beta_{1-42}$ overexpression in their glutamatergic neurons exhibited significant reduction in glutamatergic neurons in comparison to controls, implicating $A \beta$-mediated neurodegeneration of glutamatergic neurons in the models of AD (Fig. 2e-f). PEP administration almost completely annulled A $\beta$-induced neurodegeneration (Fig. 2e-f).

Encouraged by the strong in vivo glutamatergic neuronal protection by PEP, we asked whether this benefit is preserved in mammalian cells including in the HT-22 and SH-SY5Y cells. To this end, mammalian HT22 mouse hiPEPocampal cells (undiffentiated), which are devoid of cholinergic and glutamate receptors, were utilized to evaluate glutamate-induced cell death and examine the neuroprotective effect of PEP via checking cell viability using the MTT assay. Glutamate at $5 \mathrm{mM}$ showed a significant toxicity (around $45 \%$ ) in the HT-22 cells compared to vehicle control (Fig. 2g-j). Exposing the HT-22 cells to PEP extract as co-treatment with glutamate resulted in a dose-depended inhibition of HT-22 cell death (Fig. $2 \mathrm{~g}$ ).

Furthermore, $6 \mathrm{~h}$ - and $12 \mathrm{~h}$-pretreatment with PEP showed even better cell protection against glutamate toxicity ( $5 \mathrm{mM}, 24 \mathrm{~h}$ ) (Fig. 2h-i). In addition to use HT-22 cells, we further studies neuroprotective effects of PEP using the human fibroblastoma SH-SY5Y cells. We used the two-step retinoic acid (RA) and brainderived neurotrophic factor (BDNF) protocol and successfully differentiate the SH-SY5Y cells to neuronallike cells (Supp. Fig. $1 \mathrm{~b}-\mathrm{d}$ ), followed by $A \beta$ toxicity assay. $A \beta_{25-35}$ peptides reduced cell viability in dosedependent manners compared to vehicle control (Supp. Fig. 1e). Co-administration of PEP at $100 \mu \mathrm{g} / \mathrm{ml}$ (but not lower doses as we tested), with $A \beta_{25-35}$ or as pre-treatment at 6 hours (but not 12 hours) prior to $A \beta_{25-35}$ administration, was sufficient to protect against $A \beta_{25-35}$-induced neuronal death (Fig. 3a-c). Our data suggest PEP protects against $A \beta_{25-35}$-induced cellular death in both mouse and human neuronallike cells.

P. edulis extract increased neuronal mitophagy in human neurons and C. elegans 
Compromised mitophagy-induced accumulation of damaged mitochondria in the brain, especially in the entorhinal cortex and the hippocampus, is an early sign and a risk factor of $A D[8,10,27]$. Our previous studies show that genetic or pharmacological restoring of neuronal mitophagy abrogated memory loss and pathologies in AD [10,27]. Here, we asked whether PEP could induce mitophagy, and if yes, whether PEP-induced memory retention is dependent on mitophagy activation. Mitophagy is a sub-type of selective autophagy, thus there are many proteins participating in both cellular events [43,44]. For mechanistic exploration, we checked expression levels of proteins critical for the mitophagy and autophagy pathways using the SH-SY5Y-differentiated neuronal-like cells. Immunoblot data showed that PEP $(100 \mu \mathrm{g} / \mathrm{mL}$, 6h pre-treatment) inhibited phosphorylation of the mammalian target of rapamycin (mTOR) (Fig. 3d, e), reduced phosphorylation of ULK1 at p-S757 (activation of this site inhibits ULK1 activity), and increased the expression of the lysosome protein Cathepsin D; compared with the A $\beta_{25-35}$

group, $A \beta_{25-35}+$ PEP did not have significant effects on the protein levels of PINK1, Parkin, p62, SOD-1 or SOD2 (Fig. 3d-i).

While the immunoblot data strongly suggest a possibility that PEP affects mitophagy/autophagy proteins, we further designed experiments to validate this possibility. To investigate that PEP could induce mitophagy in neurons, we utilized two composite systems for monitoring mitophagy in vivo [45, 46]. First, we utilized transgenic animals expressing a mitochondria-targeted GFP together with the autophagosomal marker LC3/LGG-1 fused with DsRed [10, 47]. Normally, mitophagy-inducing stimuli encourage the formation of autophagosomes that extensively co-localize with mitochondria [46]. Here, we demonstrate a pronounced induction of mitophagy via formation of autophagosomes consisting of mitochondria upon PEP exposure (Fig. 4a-b). This implies that PEP was able to promote the formation of mito-autophagsosmes for mitochondria cargo for degradation via mitophagy. Next, we wanted to establish whether the mitochondria in the autophagosome were indeed degraded. For this purpose, we utilized transgenic animals expressing mitochondria-targeted Rosella (mtRosella) biosensor that combines a fast-maturing $\mathrm{pH}$-insensitive DsRed fused to a pH-sensitive green fluorescent protein (GFP) variant [48]. Mechanistically, quenching of the GFP signal upon uptake of the mitochondrial cargo by the acidic lysosome, is indicated by a lower GFP/DsRed ratio representing mitophagy stimulation [46]. PEP was indeed able to stimulate mitophagy as the mtRosella animals displayed significantly decreased GFP/DsRed ratio compared to vehicle controls (Fig. 4c-d). Combined the human cell data and the nematode data, we propose PEP stimulates mitophagy via activating the key mitophagy/autophagy protein ULK1 and increasing the expression of lysosome protein Cathepsin D.

\section{P. edulis extract promotes mitochondrial homeostasis and oxidative resistance via DAF-16 nuclear} translocation in C. elegans

In addition to the mechanism mentioned above, we wondered whether PEP-based neuronal benefits could be started at transcriptional level. We used Real-time PCR and checked the mRNA levels of a list of genes in the groups of 'mitophagy', 'mitochondrial unfolded protein response (UPR ${ }^{\mathrm{mt}}$ )', and 'oxidative stress', which are linked to neuroprotection $[49,50]$. Surprisingly, PEP did not induce significant change, despite an upward trend in genes associated with mitophagy (i.e., $p d r-1, d c t-1, \operatorname{lgg}-1$, and $s k n-1$ ) in the hA $\beta_{1-42}$ 
model of AD and in the WT N2 (Fig. 5a-b). This could be due to technical limitation as the whole nematode tissue was used for RNA extraction while the cells we were interested in, the total neurons (302 neurons), only constitutes around $10 \%$ of the total cells in a hermaphrodite nematode. However, significant upregulation of genes associated with oxidative stress (gst-4 and sod-3) as well as the mitochondrial unfolded protein response (UPR ${ }^{\mathrm{mt}}$ ) (ubl-5) were observed in the N2 animals (Fig. 5a). Whilst the $h A \beta_{1-42}$ model of $A D$ exhibited upregulation of only sod-3 upon PEP application (Fig. 5b). SOD3 has a role in suppressing oxidative stress that underlies mitochondrial and cellular dysfunction [51]. Previous studies reported that DAF-16 (ortholog for the mammalian FOXO transcription factors) is the upstream regulator of gst-4 and sod-3 $[52,53]$. In our nematode system, the expression level of daf-16 gene was not changed in either the N2 controls or the hA $\beta_{1-42}$ model of AD (Fig. 5a-b). Therefore, we went on to investigate whether PEP supplementation could promote the nuclear translocation of DAF-16 by using a transgenic nematode with a DAF-16::GFP-tag. A 4-point grading system was utilized for characterizing DAF-16 localization from the solely in the cytosol (1) to predominant nuclear localization (4) (Fig. 5c-d). Under physiological conditions, DAF-16 was distributed predominantly in the cytoplasm; however, upon stimulation with heat-shock (a positive control), DAF-16 was mainly localized in the nucleus (Fig. 5c). PEP induced significant nuclear translocation of DAF-16 (Fig. 5c and e). Altogether, our data suggest that PEP upregulates $g s t-4$ and sod-3 genes via enhancing subcellular distribution of DAF16 from the cytoplasm to the nucleus, resulting in increased DAF-16-regulated transcription activity.

\section{P. edulis extract protects against A $\beta$-induced memory loss is daf-16 dependent}

To further investigate whether the neuroprotective effect of PEP is daf-16 dependent or not, we knocked down the daf-16 or sod-3 gene by using RNAi feeding of the animals from egg hatching. $\mathrm{N} 2^{\text {neu-sid} 1}$ and $\mathrm{hA} \beta_{1-42}(\mathrm{JKM} 2)^{\text {neu-sid1 }}$ transgenic animals were used in these experiments, and our results suggested that knock down of daf-16 gene expression not only caused memory deficits in healthy control $\mathrm{N} 2^{\text {neu-sid}} 1$ animals, but also abolished memory restoration ability of PEP extract in both $\mathrm{N} 2{ }^{\text {neu-sid} 1}$ and $h A \beta_{1-42}(J K M 2){ }^{\text {neu-sid1 }}$ animals. However, sod-3 RNAi only abolished memory restoration of PEP in $h A \beta_{1-42}(J K M 2){ }^{\text {neu-sid1 }}$ animals but had no effect in healthy control N2 ${ }^{\text {neu-sid1 }}$ animals (Fig. $\left.5 f, g\right)$. Collectively, PEP inhibited memory deficits through upregulation of the DAF16-SOD3 pathway.

\section{Identification of potential bioactive compounds in P. edulis pericarp}

The neuroprotective effect of PEP could be contributed by the small bioactive compounds inside the extracts. To identify small molecules in PEP, we used Gas chromatography-mass spectrometry (GC-MS). Over hundreds of compounds have been identified in PEP extract and the list of compounds was shown in supplementary Table 2. To further narrow down the list of potential candidates which might inhibit AD pathologies and own translational potential, we considered capacity of compound candidates to pass the blood-brain-barrier (BBB) $[54,55]$. We used the SwissADME software to predict BBB permeability of all compounds. As a result, 15 compounds were highly ranked with BBB permeability (Table.1). While the compound phenol showed the highest BBB permeability score in this system, others such as squalene, 
tocopherols, amyrins may have high affinity to BBB receptor(s) in the BBB permeant system. Since PEP extract enhanced DAF-16 nuclear localization, a computer docking analysis was used to predict whether PEP extract containing potential compounds that could induce FOXO3/DAF-16 nuclear translocation in different conditions, including via inhibiting the insulin/IGF-1 signaling pathway. $2 \mathrm{KJI}$, an insulin-like protein found in $C$. elegans was used as a target protein, and the docking analysis was performed on the top 10 potential compounds in the list. For the results, a higher negative binding energy indicates a higher stability of the protein-ligand complex. In our study (Supplementary Table 3), tocopherols including atocopherol $(-8.556 \mathrm{kcal} / \mathrm{mol})$, Y-tocopherol $(-8.356 \mathrm{kcal} / \mathrm{mol})$, and $\delta$-tocopherol $(-8.227 \mathrm{kcal} / \mathrm{mol})$ showed the highest binding potential to the insulin like protein in $C$. elegans. Other compounds such as stigmast4-en-3-one $(-8.246 \mathrm{kcal} / \mathrm{mol})$, squalene $(-8.186 \mathrm{kcal} / \mathrm{mol})$ and cholest-4-en-3-one $(-8.151 \mathrm{kcal} / \mathrm{mol}), \mathrm{a}$ amyrin $(-7.874 \mathrm{kcal} / \mathrm{mol})$, as well as $\beta$-amyrin $(-7.453 \mathrm{kcal} / \mathrm{mol})$ could form a stable complex with $2 \mathrm{KJl}$; these data suggest that attenuation of the insulin pathway via these compounds may activate DAF-16 nuclear translocation. Further wet laboratory experiments are necessary to identify the compound(s) that could induce mitophagy and forestall memory loss and attenuate pathologies in AD animals.

\section{Discussion And Conclusion}

Where there is no drug available to cure $A D$, turning up mitophagy is suggested as a promising strategy for anti-AD drug development $[10,27,56]$. Here, we demonstrate that $P$. edulis pericarp extract not only alleviated neurodegeneration but also inhibited memory impairment in AD C. elegans, especially in the $h A \beta_{1-42}$ model of $A D$. In particular, we show these benefits to be mediated by the nuclear localization of DAF-16, which stimulates mitophagy and protects against oxidative stress. FOXO3/DAF-16 is a fundamental component of the insulin/IGF signaling (IIS) pathway, which plays a critical role in longevity and stress resistance in various organisms including in humans [57-60]. In C. elegans, DAF-16 not only regulates longevity and dauer development, but is also involved in metabolism and stress resistance. The activity of DAF-16 is regulated by the upstream protein, DAF-2 (orthologue of the mammalian insulin and insulin-like growth facor-1 receptor) [61, 62]. Upon activation, DAF-16 disconnects from the 14-3-3 proteins that negatively regulates the insulin-like signaling (IIS) pathway and is positively regulated by the JNK pathway [63]. Upon translocation to the nucleus, DAF-16 promotes target genes expression of transmembrane tyrosine kinase (old-1) [64], glutathione-S-Transferase 4 (gst4) [65, 66], NIX/dct-1 [48, 67], and sod-3 $[68,69]$.

Here, we show that the DAF-16-regulated downstream genes, including gst-4 and sod-3, were increased upon PEP supplementation. In line with this, our results showed that PEP increased DAF-16 nuclear translocation. In turn, activated DAF-16 directly promotes sod-3 overexpression level. To note, although enhanced DAF-16 activity, we did not detect significant change of NIX/dct-1; this could be caused by the use of whole worm tissue for the PCR rather than to use the isolated neurons. Related experiments will be performed using a neuronal isolation protocol for tissue collection in the future. Interestingly, our immunoblot data show that PEP increased activity of ULK1, a fundamental protein involved in autophagy initiation and mitophagy execution $[43,70]$. In line with this, two in vivo mitophagy quantification assays 
unambiguously validate neuronal mitophagy induction capacity by PEP. Taking all the pieces of data together, it suggests that PEP could regulate both mitophagy and mitochondrial resilience, which may contribute to memory retention and neuroprotection in the AD animals (Fig. 6).

Until here, the neuroprotective effects and underlying mechanism of PEP extract has been partially uncovered in this study. By extrapolation, it is likely that multiple small compounds in PEP that contributed to the beneficial effects. Fortunately, our findings clearly show $P$. edulis pericarp could be a good source of bioactive compounds, and potential compounds of PEP extract were identified in this study. In the future, it will be interesting to continue studying the therapeutic ability of the potential candidates in multiple AD models.

\section{Materials And Methods}

\section{Chemicals}

Dulbecco's modified Eagle medium (DMEM), fetal bovine serum (FBS), penicillin-streptomycin, petroleum ether, Retinoic acid (RA), Thiazolyl Blue Tetrazolium Bromide as well as Ampicillin were purchased from Sigma-Aldrich. DEME/F-12 (1:1) (1X) + GlutaMAX ${ }^{\mathrm{TM}}$-|Dulbecco's Modified Eagle Medium F-12 Nutrient Mixture (Ham) was purchased from gibco ${ }^{\circledR}$ by life technologies ${ }^{T M}$. Dimethyl sulfoxide (DMSO) and Isopropylthio-ß-galactoside (IPTG) were purchased from Merck. The CytoTox 96® LDH kit was purchased from Promega. $\beta$-Amyloid (25-35) and BDNF (human) were purchased from GenScript ${ }^{\circledR}$. TRIzol Reagent ( (Cat. \#BCCD4264) was purchased from Sigma ${ }^{\circledR}$ Life science. NuPAGE ${ }^{T M} 4-12 \%$ Bis-Tris Gels were purchased from invitrogen by Thermo Fisher Scientific. PowerSYBR® Green PCR Master Mix was purchased from aPEPlied biosystems by Thermo Fisher Scientific. NuPAGE ${ }^{\circ}$ MES SDS Running Buffer (20X) and NuPAGE ${ }^{\circledR}$ Transfer Buffer (20X) were purchased from Invitrogen by Life Technologies ${ }^{\mathrm{TM}}$ and Nover ${ }^{\circledR}$ by Life Technologies ${ }^{T M}$, respectively. Immun-Blot ${ }^{\circledR}$ PVDF Membranes for Protein Blotting (Cat. \#1620177) and iScript cDNA Synthesis kit (Cat. \#1708891) were purchased from BIO-RAD. Additionally, nonfat dry milk, antibodies including mTOR (7C10), ULK1 (D9D7), p-ULK1 (ser757) (D706U), SQSTM1/p62 (\#5114), parkin [PRK8], and GAPDH (14C10) as well as anti-rabbit lgG HRP-linked antibody and anti-mouse IgG HRP-linked antibody were purchased from Cell Signaling. Antibodies including $\mathrm{p}$ mTOR (ab109286), pink1 (38CT18.7), SOD1 (ab13498), SOD2 [2A1] (ab16956), cathepsin D [CTD-19] (ab6313), and GAP43 (ab12274) were purchased from Abcam.

\section{Plant collection and preparation}

The fresh passion fruit ( $P$. edulis 'Paul Ecke') was collected from Chiangmai, Thailand, and identified by the herbarium of Kasin Suvatabhandju (Department of Botany, Faculty of Science, Chulalongkorn University, Thailand) with the voucher specimen [016437 B(CU)]. Pericarp was cut and air-dried before being ground into a fine powder. P. edulis pericarp powder was macerate in petroleum ether with a ratio of 1: 10. The PEP extract was filtered through Whatman No.1 filter paper and concentrated using vacuum distillation and a rotary evaporator. Stock solution was prepared in DMSO (concentration is $100 \mathrm{mg} / \mathrm{mL}$ ). 


\section{Cell culture}

HT-22, a mouse hippocampal cells (a gift from Professor David Schubert, San Diego, CA, USA) were cultured and maintained in DMEM with 10\% FBS supplementary and 1\% penicillin-streptomycin. SHSY5Y, a human fibro-blastoma cells were cultured and maintained in DNEM/F12 with 10\% FBS supplementary and $1 \%$ penicillin-streptomycin (normal culture medium). Cells were maintained in the humidified incubator $\left(37^{\circ} \mathrm{C}, 5 \% \mathrm{CO}_{2}\right)$. Culture medium was changed every 2 to 3 days and 80 to $90 \%$ confluence cells were used for future experiments.

\section{Cell differentiation}

SHSY5Y cells differentiation was induced using a previously described protocol with slight modifications[71]. Briefly, at Day 0, SHSY5Y cells were seeded into the experimental plates with normal culture medium and cultured overnight. On Day1, replace the normal culture medium by using DMEM/F12 medium (5\% FBS) with retinoic acid (RA) $(10 \mu \mathrm{M})$ to initiate cell differentiation, and change to DMEM/F12 medium ( $2.5 \% \mathrm{FBS})$ with RA $(10 \mu \mathrm{M})$ to stimulate further cell differentiation in the Day 2 to Day 5. Additionally, DMEM/F12 medium ( $0 \% \mathrm{FBS}$ ) with $50 \mathrm{ng} / \mathrm{mL}$ brain-derived neurotrophic factor (BDNF) was used to strengthening cell differentiation in the Day 6 to Day 8. Then, the differentiated cells are ready for experiments on Day9.

\section{Cell viability}

3-(4,5-dimethylthiazol-2,5-diphenyl tetrazolium bromide (MTT), a widely used chemical indicator for cellular metabolic activity and cell viability base on the ability of nicotinamide ademine dinucleotide phosphate (NADPH)-dependent cellular oxidoreductase enzymes which reduce the yellow MTT to purple formazan in living cells. The formazan redissolved in the solubilization solution such as DMSO and provides a colorimetric assay. In this study, MTT was used to detect cell viability for both HT-22 and SHSY5Y cells. For HT-22 cells, 5,000 cells were seeded and grown in each well of 96-well plates overnight in humidified $5 \% \mathrm{CO}_{2}$ incubator at $37^{\circ} \mathrm{C}$. Next day, the cells were pre-treated $(6 \mathrm{~h}, 12 \mathrm{~h}$ and $18 \mathrm{~h})$ or cotreated with varied concentration of PEP extract $(12.5$ to $100 \mu \mathrm{g} / \mathrm{mL})$ and glutamate $(5 \mathrm{mM})$. At end up the drug treatment time, cells were exposure to MTT for $3 \mathrm{~h}$. Then, all supernatant was removed, and the formazan crystals were dissolved with DMSO $(200 \mu \mathrm{L})$ before measure the absorbance at $550 \mathrm{~nm}$. For SHSY5Y cells, the differentiated cells were used for cell viability detection. As above-mentioned, cells are ready to use after 9 days differentiation period, and pre-treated (6h, $12 \mathrm{~h}$ and $18 \mathrm{~h})$ or co-treated with varied concentration of PEP extract $(12.5$ to $100 \mu \mathrm{g} / \mathrm{mL})$ and $A \beta_{25-35}(25 \mu \mathrm{M})$. At end up the drug treatment time, cells were exposure to MTT for $3 \mathrm{~h}$. Then the synthesized formazan crystals were dissolved with DMSO $(200 \mu \mathrm{L})$ before measure the absorbance at $550 \mathrm{~nm}$.

\section{Gas chromatograph-mass spectrometry (GC-MS) and theoretical prediction of blood brain barrier (BBB) permeability of compounds analysis}


The beneficial effects of the PEP extract due to the various potential compounds. GCMS was employed to uncovering the potential drug candidates in PEP extract. PEP subfractions were sent to Scientific and Technological Research Equipment Center (STREC), Chulalongkorn University, Thailand for GC-MS analysis by using the Agilent 7890B GC system coupled with HP-5ms (part no. 19091S-433UI (30m x $0.25 \mathrm{~mm}, 0.25 \mu \mathrm{m})$ ) capillary column. The extracts were dissolved in hexane, and $1 \mu \mathrm{L}$ was injected into the column for analysis with a total 68-minute run time. Results were analyzed using MassHunter 2014 software and the potential compounds were identified by comparing with the spectral patterns with the National Institute of Standards and Technology (NIST) 2011 library. For further narrow down the potential compounds, SwissADME software (http://www.swissadme.ch) was used to analyze the BBB permeability of identified compounds, and DockThor (https://dockthor.Incc.br/v2/), an online software was used for docking analysis.

\section{Western blot}

Cell samples were collected by using $1 \mathrm{x}$ radioimmunoprecipitation assay (RIPA) buffer with protease inhibitors and phosphatase inhibitors. Protein samples were running on an NuPAGE 4-12\% Bis-Tris Protein Gel. The chemiluminescence reaction was detected using a ChemiDoc XRS System (Bio-Rad Laboratories). The following antibodies were used in this study: mTOR, p-mTOR, ULK1, p-ULK1(s757), pink1, parkin, p62, cathepsin D, SOD1, SOD2, GAP43, and GAPDH.

\section{C. elegansstrains}

C. elegans strains were maintained on Escherichia coli OP50 using the standard feeding methods. The temperature for rearing $C$. elegans was kept at $20^{\circ} \mathrm{C}$. The list of strains used in this study is shown in supplementary. Table 3.

\section{Drug treatment of $\mathrm{C}$. elegans}

The $P$. edulis extract was directly added in the melted NGM before being poured into the plates. Nematodes were treated from either egg hatching or $L 4$ stage.

\section{Toxicity assay}

A toxicity assay was used to determine the safe dose of PEP extract selection in C. elegans. N2, a wild type of strain was used for these experiments. At Day 1, ten of one day old nematodes were placed on the NGM plates with OP50 which contained PEP extract $(0.025,0.25,0.5 \mathrm{mg} / \mathrm{mL})$ or not (vehicle) for $3 \mathrm{~h}$ egg laying. The number of eggs was counted after adults were removed. On Day 2, the number of L1 larvae and unhatched eggs was counted to check the egg hatching efficiency. In the day 3 , the number of L4 larvae was counted. On Day 4, the number of one day old adult nematodes was counted. Each group includes three technical repeats.

\section{Life-span and healthy-span analysis}


$A D$ caused health issues and reduction of lifespan has been reported [1, 72]. Record the living time to check whether PEP extract having beneficial effects for lifespan extension in healthy (N2) nematodes and AD (human Tau[P301L], and human A 1 1-42) transgenic nematodes. The animals were synchronized by bleaching and grown at $20^{\circ} \mathrm{C}$ until the $\mathrm{L} 4$ stage. Twenty of $L 4$ stage animals were picked and placed in each experiment plate (with or without PEP extract). FUDR was added to prevent egg hatching and animals were transferred to the fresh plates for every 2-3 days until Day 10, and then every 5-6 days (if food running out) until death. The number of living or dead animals was recorded every day until the last animal's death. Every experiment included 3 technical repeats. Additionally, C. elegans drawing food through its pharynx, and the times of pharyngeal contraction and relaxation indicate the food uptake rate. Along with the lifespan experiment, the pharyngeal pumping rate of Day 2 and Day 8 nematodes was evaluated via manually counting for 30 seconds, and 10 animals were randomly selected from each technical repeat.

\section{Chemotaxis behavior assay}

Isoamyl alcohol (IA), a volatile liquid was employed to perform the Chemotaxis assay as previously described[73, 74]. Briefly, animals were synchronized by bleaching and grown on the OP50 seeded NGM plates with or without PEP extract at $20^{\circ} \mathrm{C}$ until day1. Animals (200 to 300 nematodes/group) were collected and washed 4 times with MilliQ water, and then placed on conditional NGM plates (no OP50) with/ without IA on the middle of lid $(10 \mu \mathrm{L})$ for $90 \mathrm{~min}$. After that, animals were washed and transferred to the start point in the experimental plates and the number of animals from each area was recorded after $2 \mathrm{hrs}$. The experimental plate $(10 \mathrm{~mm})$ separates to three main areas, which were labeled as $I A, T$ and $S$ (start point). A small piece of Parafilm was placed in the middle of 'IA' area and $3 \mu \mathrm{L}$ of $2 \% \mathrm{IA}$ was toPEPed on the Parafilm. The chemotaxis index was calculated as ('IA' - 'T') / ('IA' + 'T' + 'S').

\section{mRNA quantification in C. elegans tissue}

Target gene expression in $C$. elegans was determined using real-time PCR. Animals were synchronized by bleaching and grown on the OP50 seeded NGM plates with or without PEP extract at $20^{\circ} \mathrm{C}$ until day 1 . Nematodes were collected, and then total RNA was further isolated using TRIzol ${ }^{\mathrm{TM}}$ reagent. The concentration of RNA was detected using Nano-drop machine under absorbance $260 \mathrm{~nm}$. cDNA samples were prepared using the iScript ${ }^{\mathrm{TM}} \mathrm{CDNA}$ synthesis kit, and synthesis for $5 \mathrm{~min}$ at $25^{\circ} \mathrm{C}, 20$ min at $46^{\circ} \mathrm{C}, 1$ min at $95^{\circ} \mathrm{C}$, and finishing at $4^{\circ} \mathrm{C}$. The synthesized $\mathrm{cDNA}$ samples were used for real-time quantitative reverse transcription PCR (RT-qPCR) for target gene expression quantification. A total of nine samples for three biological repeats (three technical repeats for each biological repeat) were used. PowerSYBR ${ }^{\circledR}$ Green PCR Master Mix was employed for quantitative PCR analysis. For each reaction, $4 \mu \mathrm{L}$ of cDNA temple was mixed with $2 x$ PowerSYBR ${ }^{\circledR}$ Green PCR Master Mix $(5 \mu \mathrm{L})$, forward primer $(0.5 \mu \mathrm{L})$, reverse primer $(0.5 \mu \mathrm{L})$ in total $10 \mu \mathrm{L}$. Real-time qPCR reactions were performed in QuantStudio ${ }^{\mathrm{TM}} 7$ Flex System v1.1 (applied biosystems by Life Technologies). The thermal cycling condition was set as predenaturation step at $95^{\circ} \mathrm{C}$ for 10 min, which followed by 40 cycles of denaturation at $95^{\circ} \mathrm{C}$ for $15 \mathrm{~s}$, 
annealing at $60{ }^{\circ} \mathrm{C}$ for $1 \mathrm{~min}$, and extension at $95^{\circ} \mathrm{C}$ for $15 \mathrm{~s}$ and $60^{\circ} \mathrm{C}$ for $1 \mathrm{~min}$. A melting curve was per-formed to confirm product formation. Data was calculated by using the $2^{-\triangle \Delta C T}$ method. The primers used in this study was listed as following:

rheh-1, 5'-GGCTCCAACCTTACCACTCC-3' and 5'-GCAAATCCTACT GCTGCTCC-3', unc-51, 5'-CTACACGTGGTGACTCTCCG-3' and 5'-ATGCAATACGACGCGAAAGC-3' pink1,5'-AGCATATCGAATCGCAAATGAGTTAG-3' and 5'-TCGACCGTGGCGAGTTACAAG-3', pdr-1, 5'-AGCCACCGAGCGATTGATTGC-3' and 5'-GTGGCATTTTGGGCATCTTCTTG-3', dct-1, 5'-GGCTCCAACCTTACCACTCC-3' and 5'-GCAAATCCTACT GCTGCTCC-3', Igg-1, 5'-ACATGATTTTCTGGGAGGGG-3' and 5'-TCTAATGGAAACCCAAAGCC-3', skn-1, 5'-ACAGTGCTTCTCTTCGGTAGC-3' and 5'-GAGACCCATTGGACGGTTGA-3', daf-16, 5'-AAGCCGATTAAGACGGAACC-3' and 5'-GTAGTGGCATTGGCTTGAAG-3', hsp6, 5'-AACCACCGTCAACAACGCCG-3' and 5'-AGCGATGATCTTATCTCCAGCGTCC-3', hsp4, 5'-GCACTGGCCGTTCAAGATCGTCG-3' and 5'-TGCTGGCACGGTGACAACGG-3', ubl5, 5'-ACAAACTGGAACACGATGGGA-3' and 5'-TCCCTCGTGAATCTCGTAATCC-3', hsf-1, 5'-GAATGCGACTAGGCAAATGGC-3' and 5'-GGTGGATGAGGTGGAAGTCG-3', gst-4, 5'-TGCTCTTGCTGAGCCAATCCGT-3' and 5'-CCAGCGAGTCCAAATTTTCTTGCCA-3', sod-1, 5'-CCGACACGCTCGTCACGCTT-3' and 5'-ACTGGGGAGCAGCGAGAGCA-3', sod-2, 5'-ACAGGAGTCGCTGCTGTTCGC-3' and 5'-TCCTTTGGAGACCGCCTCGTGA-3', sod-3, 5'-CTAAGGATGGTGAACCTTCA-3' and 5'-CGCGCTTAATAGTGTCCATCAG-3', pmp3, 5'-ATGATAAATCAGCGTCCCGAC-3' and 5'-TTGCAACGAGAGCAACTGAAC-3'.

\section{Aldicarb assay}

Aldicarb, an acetylcholinesterase inhibitor was employed to evaluate the sensitivity of $C$. elegans to the synaptic transmission of acetylcholine at the neuromuscular junction. The $C$. elegans strains VC233 and NM204 grown on the OP50 seeded NGM plates were used as hypersensitive and resistant control in the experiment, respectively. Animals for experiments were synchronized by bleaching and grown on the OP50 seeded NGM plates with or without PEP extract at $20^{\circ} \mathrm{C}$ until day 1 . Thirty animals were transferred on each NGM plate with $0.75 \mathrm{mM}$ aldicarb, and the non-paralyzed animals were recorded every $30 \mathrm{~min}$ for 
the aldicarb-induced paralysis. Each experimental group includes 3 biological repeats and 3 technical repeats.

\section{Glutamatergic neurons imaging}

Glutamatergic neurodegeneration was detected in C. elegans using the previous reported method. Animals were synchronized by bleaching and grown on the OP50 seeded NGM plates with or without PEP extract at $20^{\circ} \mathrm{C}$ until adult day3. There are about 15 glutamatergic neurons in the worm tail region, and 5 were selected in this study, which are $L U A(R), L U A(L), P V R, P L M(R)$, and PLM $(L)$. The tail regions of day 3 animals were imaged using confocal microscope. Each experimental group includes 2 biological repeats and 3 technical repeats.

\section{Screening of neuronal mitophagy in $\mathrm{C}$. elegans}

Two $C$. elegans strains were employed to quantify mitophagy induction potential of PEP extract in C.

elegans. For both experiments, the nematodes were prepared and placed on the OP50 seeded NGM plates with or without PEP extract $(250 \mu \mathrm{g} / \mathrm{mL})$ from egg hatching stage. The first transgenic animal expressing LGG-1::DsRed (autophagosomal marker), together with DCT-1::GFP (mitophagy reporter) in neurons. The double positive animals (adult day 1 ) were paralyzed by levamisole, mounted on $4 \%$ agarose pads, and imaged using confocal microscopy. The co-localization of LGG-1 and DCT-1 was count for mitophagy events. Another transgenic animal expressing pan-neuronal mitophagy reporter (mt-Rosella biosensor), which represent the mitophagy level according to the ratio between $\mathrm{pH}$-sensitive GFP to $\mathrm{pH}$-insensitive DsRed (the lower ratio, the higher mitophagy events). The day 1 animals were paralyzed by levamisole, mounted on $4 \%$ agarose pads, and imaged using confocal microscope at $10 x$ or $40 x$ magnifications. Each experimental group includes 2 biological repeats and 3 technical repeats.

\section{DAF-16 nuclear localization}

Animals were synchronized by bleaching and grown on the OP50 seeded NGM plates at $20^{\circ} \mathrm{C}$ until L4 stage. Animals were transferred to the OP50 seeded NGM plates with or without PEP extract for $24 \mathrm{~h}$. The DAF-16::GFP nuclear translocation of the adult day1 nematodes were paralyzed by levamisole, mounted on $4 \%$ agarose pads, and imaged using confocal microscope at $40 x$ magnifications. Each experimental group includes 2 biological repeats and 2 technical repeats. The nuclear localization level was scored as Level1 to Level4.

\section{RNA interference (RNAi) by feeding}

Feeding bacteria expressing dsRNA (feeding RNAi) was used to knock-down selected targets (daf-16 and sod-3) in C. elegans. Briefly, selected bacteria were grown in the LB (50 mg/mL Ampicilin) and added on the NGM plates containing 1\% Ampicillin and 1\% IPTG with/without PEP extract. For experiments, the animals were synchronized by bleaching and grown on these RNAi plates until Adult Day1.

\section{Statistical analysis}


All results presented in this study have at least two biological repeats, except lifespan (one biological repeat with three technical repeats). For the imaging base experiments, data were quantified using Image J software. And statistical data analyzed using Prism 8.0 software. The data were presented in mean \pm SEM. The difference between two treatment groups was analyzed using unpaired t-tests. And the group differences were analyzed using one-way ANOVA with Tukey's multiple comparisons test. The difference for multiple targets was analysis using two-way ANOVA with Sidak's multiple comparisons test. $\mathrm{P}<0.05$ considered as statistically significant.

\section{Declarations}

\section{Funding}

S.Q.C is a Ph.D. student in Clinical Biochemistry and Molecular Medicine, Department of Clinical Chemistry, Faculty of Allied Health Sciences, Chulalongkorn University, Thailand, and a visiting Ph.D. student in the Department of Clinical Molecular Biology, University of Oslo and Akershus University Hospital Norway. Her study was financially suPEPorted by the scholarship from the Graduate School, Chulalongkorn University to commemorate $72^{\text {nd }}$ Anniversary of his Majesty King Bhumibol Adulyadej and the $100^{\text {th }}$ Anniversary Chulalongkorn University Fund for Doctoral Scholarship as well as the $90^{\text {th }}$ Anniversary Chulalongkorn University Fund (Ratchadaphiseksomphot Endowment Fund) and Overseas Research Experience scholarship for Graduate student. E.F.F. was supported by HELSE S ØR-ØST (\#2017056, \#2020001, \#2021021), the Research Council of Norway (\#262175), the National Natural Science Foundation of China (\#81971327), Akershus University Hospital (\#269901, \#261973), the Civitan Norges Forskningsfond for Alzheimers sykdom (\#281931), the Czech Republic-Norway KAPEPA programme (with Martin Vyhnálek, \#T001000215), and the Rosa sløyfe/Norwegian Cancer Society \& Norwegian Breast Cancer Society (\#207819).

\section{Acknowledgements}

We would like to thanks Thale Dawn Patrick-Brown for the English language editing.

\section{Author Contributions}

T.T and E.F.F conceptualized and supervised the study. E.F.F managed the research project, provide resources as well as evaluated methodologies for the study. S.Q.C and Y. A performed the experiments and data analysis. T.T and E.F.F validated the experimental results. S.Q.C and Y. A wrote the first draft of the manuscript. T.T and E.F.F revised the manuscript, and all approved the final manuscript.

\section{Declaration of Interests}

E.F.F. has CRADA arrangement with ChromaDex, and is consultant to Aladdin Healthcare Technologies, Vancouver Dementia Prevention Centre, and Intellectual Labs.

Ethics approval Not applicable, and no human researches were involved in this study. 
Consent to participate Not applicable.

Consent for publication All authors have seen and approved the manuscript and contributed significantly to this work.

\section{Data availability statement}

All data are in the manuscript and the associated supporting information file.

\section{References}

1. Wiley J (2021) Alzheimer's disease facts and figures. Alzheimers Dement 17:327-406

2. Kumar A et al (2021) Alzheimer Disease (Nursing).

3. Alonso AdC, Grundke-lqbal I, lqbal K (1996) Alzheimer's disease hyperphosphorylated tau sequesters normal tau into tangles of filaments and disassembles microtubules. Nat Med 2(7):783-787

4. Chen $\mathrm{G}$ et al (2000) A learning deficit related to age and $\beta$-amyloid plaques in a mouse model of Alzheimer's disease. Nature 408(6815):975-979

5. Goedert M et al (1989) Multiple isoforms of human microtubule-associated protein tau: sequences and localization in neurofibrillary tangles of Alzheimer's disease. Neuron 3(4):519-526

6. Hardy JA, Higgins GA (1992) Alzheimer's disease: the amyloid cascade hypothesis. Science 256(5054):184-186

7. Zhang Y et al (2021) Amyloid-beta toxicity modulates tau phosphorylation through the PAX6 signalling pathway.Brain,

8. Kobro-Flatmoen A et al (2021) Re-emphasizing early Alzheimer's disease pathology starting in select entorhinal neurons, with a special focus on mitophagy. Ageing Res Rev 67:101307

9. Kerr JS et al (2017) Mitophagy and Alzheimer's Disease: Cellular and Molecular Mechanisms. Trends Neurosci 40(3):151-166

10. Fang EF et al (2019) Mitophagy inhibits amyloid-beta and tau pathology and reverses cognitive deficits in models of Alzheimer's disease. Nat Neurosci 22(3):401-412

11. Canter RG, Penney J, Tsai LH (2016) The road to restoring neural circuits for the treatment of Alzheimer's disease. Nature 539(7628):187-196

12. Zhao N et al (2020) Alzheimer's Risk Factors Age, APOE Genotype, and Sex Drive Distinct Molecular Pathways. Neuron 106(5):727-742e6

13. Xu C et al (2021) TNF-alpha-dependent neuronal necroptosis regulated in Alzheimer's disease by coordination of RIPK1-p62 complex with autophagic UVRAG. Theranostics 11(19):9452-9469

14. Aguillón-Osma J et al (2019) Impact of in vitro gastrointestinal digestion on the bioaccessibility and antioxidant capacity of bioactive compounds from Passion fruit (Passiflora edulis) leaves and juice extracts. J Food Biochem 43(7):e12879 
15. Hu Y et al (2018) A new C-glycosyl flavone and a new neolignan glycoside from Passiflora edulis Sims peel. Nat Prod Res 32(19):2312-2318

16. Pereira DTV et al (2021) Integration of pressurized liquids and ultrasound in the extraction of bioactive compounds from passion fruit rinds: Impact on phenolic yield, extraction kinetics and technical-economic evaluation, vol 67. Innovative Food Science \& Emerging Technologies, p 102549

17. Parliment TH (1972) Volatile constituents of passion fruit. J Agric Food Chem 20(5):1043-1045

18. Leão KM et al (2014) Odor potency, aroma profile and volatiles composition of cold pressed oil from industrial passion fruit residues. Ind Crops Prod 58:280-286

19. Albuquerque MACd et al (2019) Tropical fruit by-products water extracts of tropical fruit by-products as sources of soluble fibres and phenolic compounds with potential antioxidant, anti-inflammatory, and functional properties.

20. do Carmo MCL et al (2020) Passion fruit (Passiflora edulis) leaf aqueous extract ameliorates intestinal epithelial barrier dysfunction and reverts inflammatory parameters in Caco-2 cells monolayer. Food Res Int 133:109162

21. Lourith N, Kanlayavattanakul M (2020) Passion fruit seed: Its antioxidative extracts and potency in protection of skin aging, in Aging. Elsevier, pp 283-288

22. Nerdy N, Ritarwan K (2019) Hepatoprotective activity and nephroprotective activity of peel extract from three varieties of the passion fruit (Passiflora sp.) in the albino rat. Open Access Macedonian Journal of Medical Sciences 7(4):536

23. Tal $Y$ et al (2016) The neuroprotective properties of a novel variety of passion fruit. J Funct Foods 23:359-369

24. Dos Santos KC et al (2016) Passiflora actinia hydroalcoholic extract and its major constituent, isovitexin, are neuroprotective against glutamate-induced cell damage in mice hiPEPocampal slices. J Pharm Pharmacol 68(2):282-291

25. Doungue HT, Kengne APN, Kuate D (2018) Neuroprotective effect and antioxidant activity of Passiflora edulis fruit flavonoid fraction, aqueous extract, and juice in aluminum chloride-induced Alzheimer's disease rats. Nutrire 43(1):1-12

26. Perry RJ, Watson P, Hodges JR (2000) The nature and staging of attention dysfunction in early (minimal and mild) Alzheimer's disease: relationship to episodic and semantic memory impairment. Neuropsychologia 38(3):252-271

27. Xie $\mathrm{C}$ et al Amelioration of Alzheimer's disease pathology by mitophagy inducers identified via machine learning and a cross-species workflow. Nat Biomed Eng, In press

28. Gallrein $C$ et al (2021) Novel amyloid-beta pathology C. elegans model reveals distinct neurons as seeds of pathogenicity. Prog Neurobiol 198:101907

29. Cummins $\mathrm{N}$ et al (2018) Disease-associated tau impairs mitophagy by inhibiting Parkin translocation to mitochondria. EMBO J 
30. Ping $Y$ et al (2015) Linking a 342 -induced hyperexcitability to neurodegeneration, learning and motor deficits, and a shorter lifespan in an Alzheimer's model. PLoS Genet 11(3):e1005025

31. Dement A (2016) Alzheimer's disease facts and figures. Alzheimer's \& Dementia: The Journal of the Alzheimer's Association 12(4):459-509

32. Cacabelos R, Takeda M, Winblad B (1999) The glutamatergic system and neurodegeneration in dementia: preventive strategies in Alzheimer's disease. International journal of geriatric psychiatry

33. Schliebs R, Arendt $T$ (2006) The significance of the cholinergic system in the brain during aging and in Alzheimer's disease. J Neural Transm 113(11):1625-1644

34. Ferreira-Vieira H (2016) Alzheimer's disease: targeting the cholinergic system. Curr Neuropharmacol 14(1):101-115

35. Gibson GE, Peterson C, Jenden DJ (1981) Brain acetylcholine synthesis declines with senescence. Science 213(4508):674-676

36. Singla N, Dhawan D (2017) Zinc improves cognitive and neuronal dysfunction during aluminiuminduced neurodegeneration. Mol Neurobiol 54(1):406-422

37. Hampel $\mathrm{H}$ et al (2018) The cholinergic system in the pathophysiology and treatment of Alzheimer's disease. Brain 141(7):1917-1933

38. Mahoney TR, Luo S, Nonet ML (2006) Analysis of synaptic transmission in Caenorhabditis elegans using an aldicarb-sensitivity assay. Nat Protoc 1(4):1772-1777

39. Masliah E et al (1996) Deficient glutamate tranport is associated with neurodegeneration in Alzheimer's disease. Annals of Neurology: Official Journal of the American Neurological Association and the Child Neurology Society 40(5):759-766

40. Myhrer T (1998) Adverse psychological impact, glutamatergic dysfunction, and risk factors for Alzheimer's disease. Neuroscience \& Biobehavioral Reviews 23(1):131-139

41. Tu S et al (2014) Oligomeric Aß-induced synaptic dysfunction in Alzheimer's disease. Molecular neurodegeneration $9(1): 1-12$

42. Griffin EF et al (2019) ApoE-associated modulation of neuroprotection from Abeta-mediated neurodegeneration in transgenic Caenorhabditis elegans.Dis Model Mech, 12(2)

43. Aman $Y$ et al (2021) Autophagy in healthy ageing and disease. Nat Aging 1:634-650

44. Munson MJ et al (2021) GAK and PRKCD are positive regulators of PRKN-independent mitophagy. Nat Commun 12(1):6101

45. Fang EF et al (2019) Mitophagy inhibits amyloid- $\beta$ and tau pathology and reverses cognitive deficits in models of Alzheimer's disease. Nat Neurosci 22(3):401-412

46. Fang $\mathrm{EF}$ et al In vitro and in vivo detection of mitophagy in human cells, C. elegans, and mice.JoVE (Journal of Visualized Experiments), 2017(129): p.e56301

47. Fang EF et al In Vitro and In Vivo Detection of Mitophagy in Human Cells, C. Elegans, and Mice.J Vis Exp, 2017(129). 
48. Palikaras K, Lionaki E, Tavernarakis N (2015) Coordination of mitophagy and mitochondrial biogenesis during ageing in C. elegans. Nature 521(7553):525-528

49. Mouchiroud L et al (2013) The NAD(+)/Sirtuin Pathway Modulates Longevity through Activation of Mitochondrial UPR and FOXO Signaling. Cell 154(2):430-441

50. Fang EF et al (2014) Defective mitophagy in XPA via PARP-1 hyperactivation and NAD(+)/SIRT1 reduction. Cell 157(4):882-896

51. Ray A et al (2014) Mitochondrial dysfunction, oxidative stress, and neurodegeneration elicited by a bacterial metabolite in a C. elegans Parkinson's model. Cell Death Dis 5(1):e984-e984

52. Evans EA, Kawli T, Tan M-W (2008) Pseudomonas aeruginosa suPEPresses host immunity by activating the DAF-2 insulin-like signaling pathway in Caenorhabditis elegans. PLoS Pathog 4(10):e1000175

53. Henderson ST, Bonafe M, Johnson TE (2006) daf-16 protects the nematode Caenorhabditis elegans during food deprivation. The Journals of Gerontology Series A: Biological Sciences and Medical Sciences. 61:444-4605): p.

54. Van Tellingen $O$ et al (2015) Overcoming the blood-brain tumor barrier for effective glioblastoma treatment. Drug Resist Updates 19:1-12

55. Pardridge WM (2015) Blood-brain barrier endogenous transporters as therapeutic targets: a new model for small molecule CNS drug discovery. Expert Opin Ther Targets 19(8):1059-1072

56. Kingwell K (2019) Turning up mitophagy in Alzheimer disease.Nat Rev Drug Discov,

57. Barbieri M et al (2003) Insulin/IGF-I-signaling pathway: an evolutionarily conserved mechanism of longevity from yeast to humans. American Journal of Physiology-Endocrinology And Metabolism 285(5):E1064-E1071

58. Willcox BJ et al (2008) FOXO3A genotype is strongly associated with human longevity. Proc Natl Acad Sci U S A 105(37):13987-13992

59. Murphy CT et al (2003) Genes that act downstream of DAF-16 to influence the lifespan of Caenorhabditis elegans. Nature 424(6946):277-283

60. Kaletsky $\mathrm{R}$ et al (2016) The $\mathrm{C}$. elegans adult neuronal IIS/FOXO transcriptome reveals adult phenotype regulators. Nature 529(7584):92-96

61. Henderson ST, Johnson TE (2001) daf-16 integrates developmental and environmental inputs to mediate aging in the nematode Caenorhabditis elegans. Curr Biol 11(24):1975-1980

62. Kenyon $\mathrm{C}$ et al (1993) A C. elegans mutant that lives twice as long as wild type. Nature 366(6454):461-464

63. Oh SW et al (2005) JNK regulates lifespan in Caenorhabditis elegans by modulating nuclear translocation of forkhead transcription factor/DAF-16. Proceedings of the National Academy of Sciences, 102(12): p. 4494-4499

64. Murakami S, Johnson TE (2001) The OLD-1 positive regulator of longevity and stress resistance is under DAF-16 regulation in Caenorhabditis elegans. Curr Biol 11(19):1517-1523 
65. Shanmugam $G$ et al (2017) Diosgenin a phytosterol substitute for cholesterol, prolongs the lifespan and mitigates glucose toxicity via DAF-16/FOXO and GST-4 in Caenorhabditis elegans, vol 95. Biomedicine \& Pharmacotherapy, pp 1693-1703

66. Zhang L, Gu B, Wang Y (2021) Clove essential oil confers antioxidant activity and lifespan extension in C. elegans via the DAF-16/FOXO transcription factor, vol 242. Toxicology \& Pharmacology, Comparative Biochemistry and Physiology Part C, p 108938

67. Fang EF et al (2016) NAD(+) Replenishment Improves Lifespan and Healthspan in Ataxia Telangiectasia Models via Mitophagy and DNA Repair. Cell Metab 24(4):566-581

68. Honda Y, Honda S (1999) The daf-2 gene network for longevity regulates oxidative stress resistance and Mn-superoxide dismutase gene expression in Caenorhabditis elegans. FASEB J 13(11):13851393

69. Leite NR et al (2020) Baru Pulp (Dipteryx alata Vogel): Fruit from the Brazilian Savanna Protects against Oxidative Stress and Increases the Life Expectancy of Caenorhabditis elegans via SOD-3 and DAF-16. Biomolecules 10(8):1106

70. Lazarou $\mathrm{M}$ et al (2015) The ubiquitin kinase PINK1 recruits autophagy receptors to induce mitophagy. Nature 524(7565):309-314

71. Shipley MM, Mangold CA, Szpara ML Differentiation of the SH-SY5Y human neuroblastoma cell line.Journal of visualized experiments: JoVE, 2016(108).

72. Higham JP et al (2019) Alzheimer's disease associated genes ankyrin and tau cause shortened lifespan and memory loss in Drosophila. Front Cell Neurosci 13:260

73. Colbert HA, Bargmann $\mathrm{Cl}$ (1995) Odorant-specific adaptation pathways generate olfactory plasticity in C. elegans. Neuron 14(4):803-812

74. Voglis G, Tavernarakis $\mathrm{N}$ (2008) A synaptic DEG/ENaC ion channel mediates learning in C. elegans by facilitating dopamine signalling. EMBO J 27(24):3288-3299

\section{Tables}

Table 1. The SwissADME software was used for In silico predication of BBB permeability of a list of compounds identified in the $P$. edulis pericarp extract. 


\begin{tabular}{|lll|}
\hline Numbers & Name & Predicted as BBB permeant \\
\hline 1 & Phenol & Yes \\
\hline 2 & Squalene & No \\
\hline 4 & $\gamma$-tocopherol & No \\
\hline 5 & $\boldsymbol{\alpha}$-tocopherol & No \\
\hline 6 & B-amyrin & No \\
\hline 7 & O-tocopherol & No \\
\hline 8 & Cholest-4-en-3-one & No \\
\hline 9 & $\boldsymbol{\alpha}$-spinasterone & No \\
\hline 10 & Stigmast-4-en-3-one & No \\
\hline 11 & Phytol & No \\
\hline 12 & Behenic alcohol & No \\
\hline 13 & $\boldsymbol{\alpha}$-tocopherolquinone & No \\
\hline 14 & 9,19 -cyclolanost-7-en-3-ol & No \\
\hline 15 & 9,19 -cyclolanostane-3,7-diol & No \\
\hline
\end{tabular}

Figures 
a

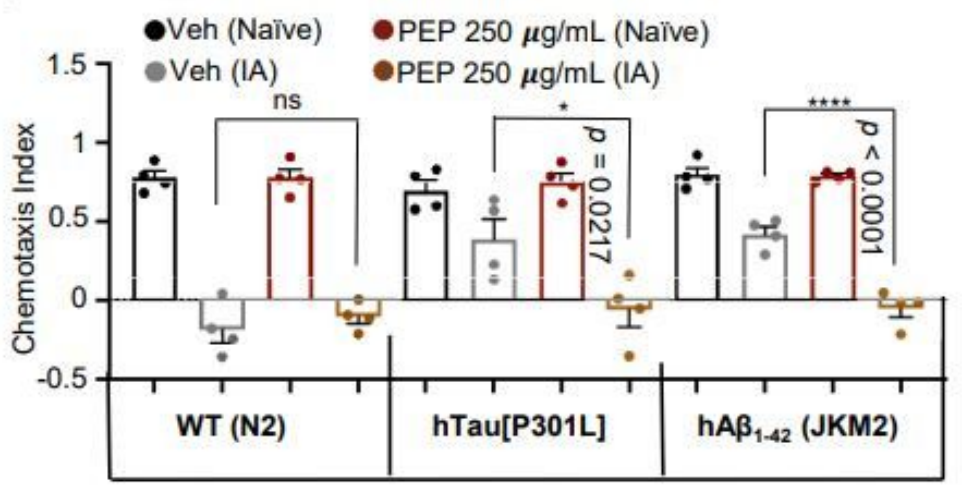

C

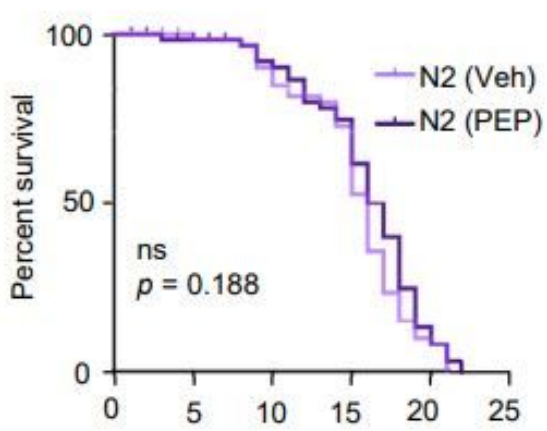

f

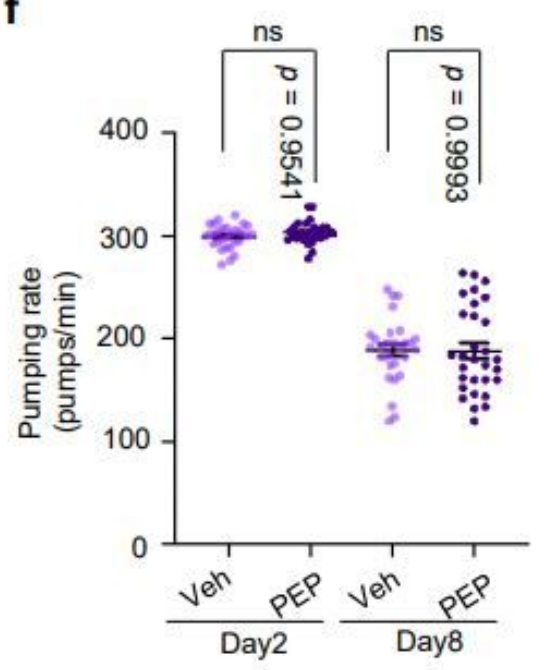

g b

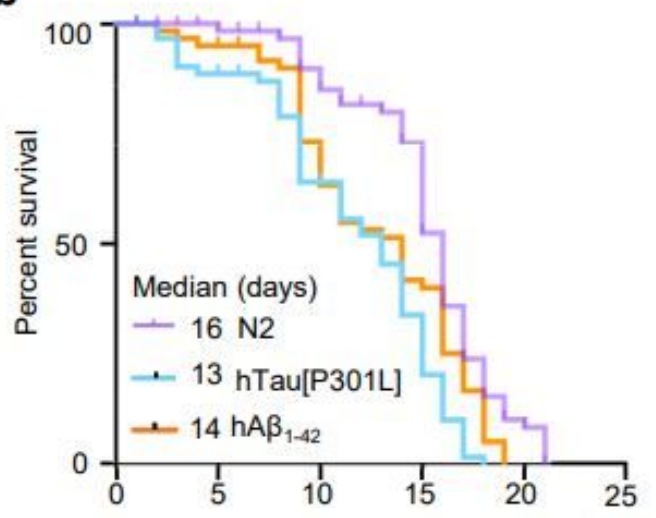

e
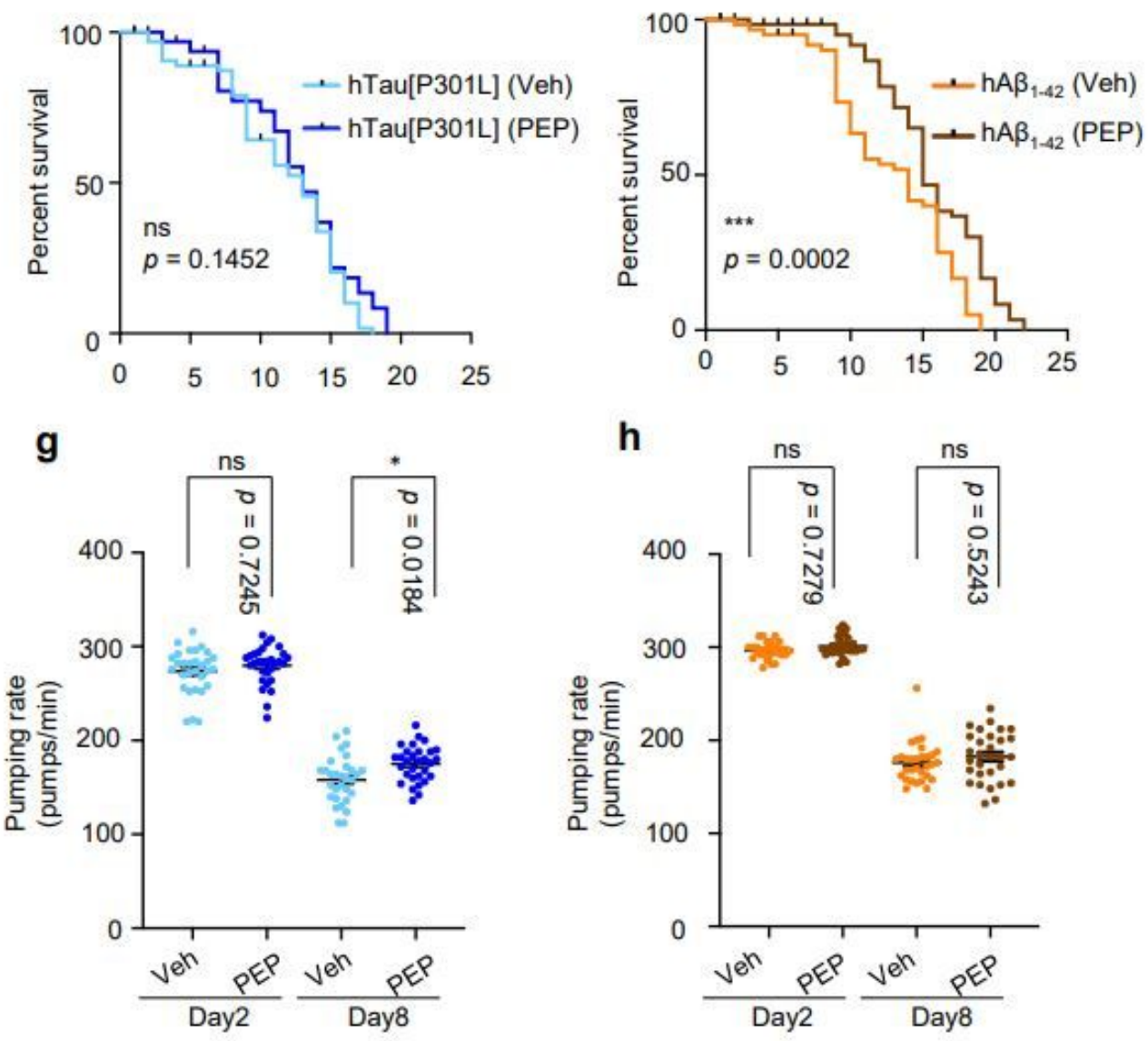

Figure 1

PEP improves memory and extends lifespan in AD models of $C$. elegans

a, PEP restored memory in adult Day-1 hTau[P301L] and hA $\beta_{1-42}(\mathrm{JKM} 2)$ C. elgans. Data were from four biological repeats with the results shown in mean \pm S.E.M. One-way ANOVA followed by Tukey's multiple comparisons test was used for data analysis with ns, no significance; ${ }^{*} p<0.05,{ }^{*} p<0.01,{ }^{* \star *} p<0.001$, $\star \star \star \star x<0.0001$. 
b, Pathological tau and $A \beta_{1-42}$ caused shorter lifespan in $h T a u[P 301 L]$, and $h A \beta_{1-42}(J K M 2)$ nematodes when compare to WT animals.

ce, PEP extended lifespan in hA $\beta_{1-42}$ (JKM2) but not WT (N2) or hTau[P301L] C. elgans. Data were from one biological repeat with three technical repeats ( $n=60$ animals). The Kaplan-Meier survival curves were presented with statistics using the Log-rank (Mantel-Cox) test: ns, no significance; ${ }^{\star} p<0.05$, ${ }^{\star \star} p<$

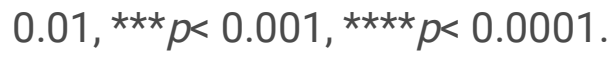

f-h, Effect of PEP on pharyngeal pumping speed in adult Day-2 and Day-8 WT (N2), hTau[P301L], and $h A \beta_{1-42}$ (JKM2) C. elegans. Data were from one biological repeat with three technical repeats. One-way ANOVA followed by Tukey's multiple comparisons test was used for data analysis with ns, no significance; ${ }^{\star} p<0.05,{ }^{*} p<0.01,{ }^{* \star *} p<0.001,{ }^{* \star \star *} p<0.0001$. 
a

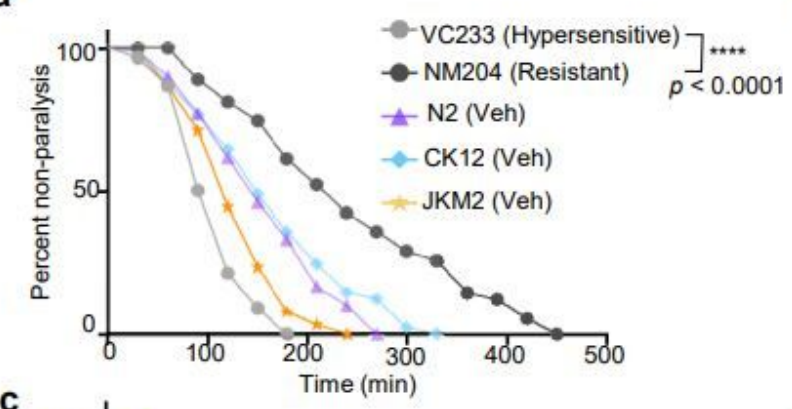

C

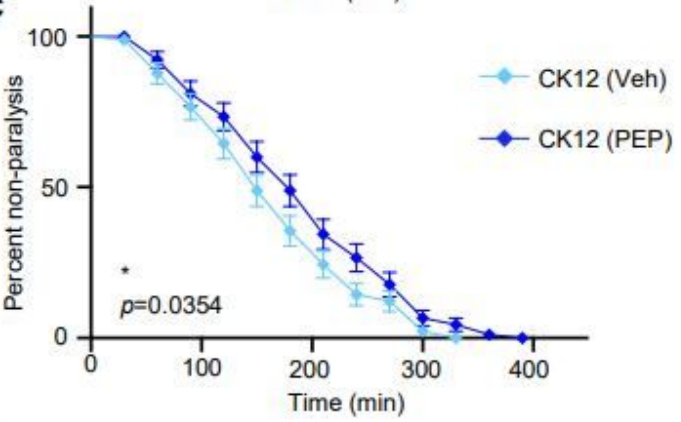

e

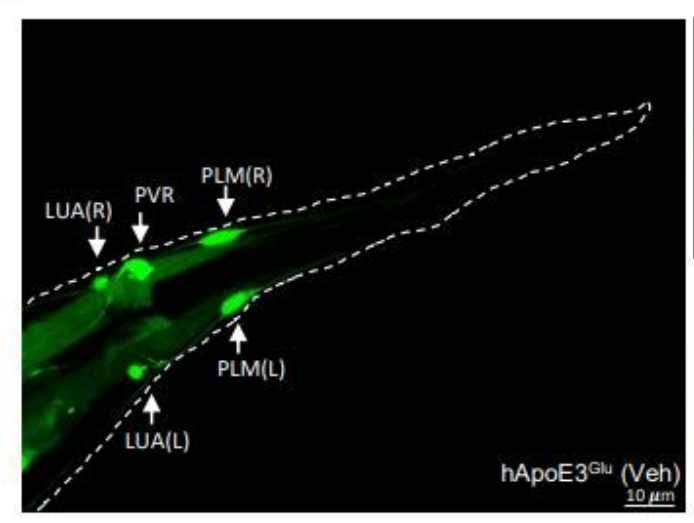

b

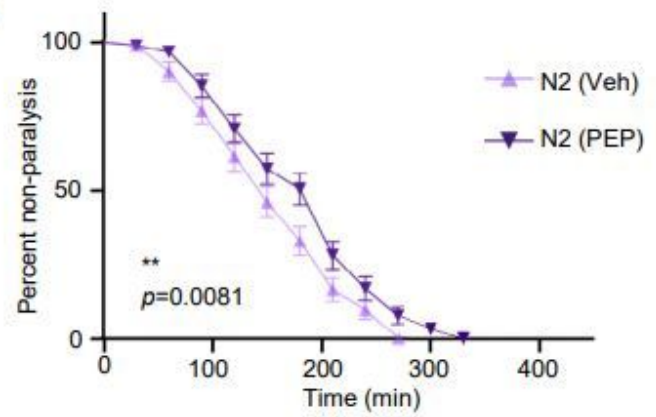

d

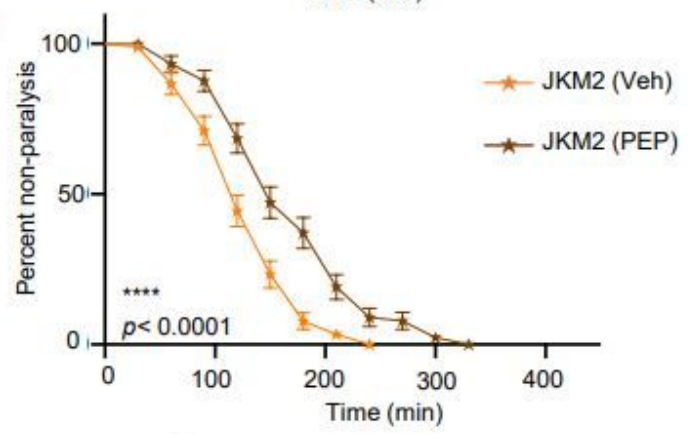

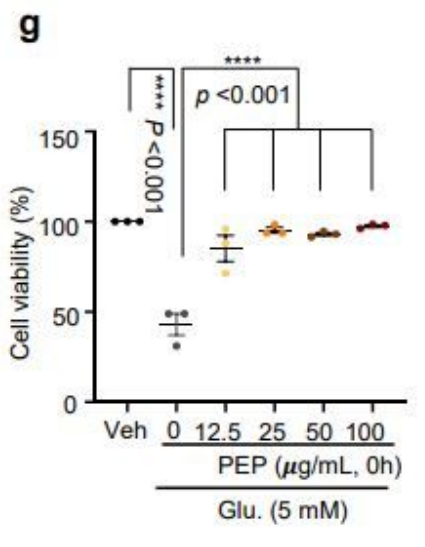
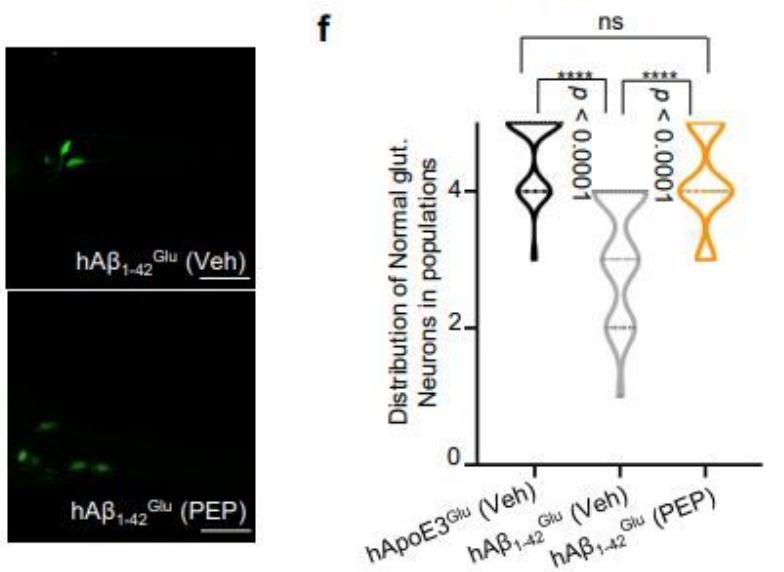

i

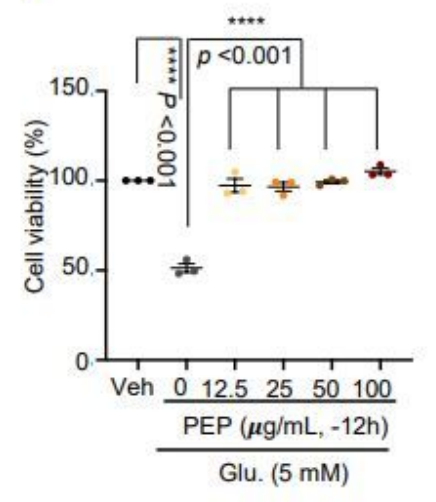

Figure 2

PEP protects cholinergic and glutamatergic neurons in the AD nematodes.

a, Nematodes reacted to the acetylcholinesterase inhibitor (Aldicarb). VC233 was an aldicarb hypersensitive strain, while NM204 was an aldicarb resistant strain. WT (N2), hTau[P301L], and hA $\beta_{1-42}$ (JKM2) nematodes were test groups. 
b-d, PEP increased neuronal resilience (as evidenced by prolonged paralysis time) against Aldicarbinduced toxicity in Day-1 WT (N2), hTau[P301L], and hA $\beta_{1-42}$ (JKM2) nematodes. Data were from three biological repeats. Log-rank (Mantel-Cox) test was used for data analysis with ns, no significance; * $p<$

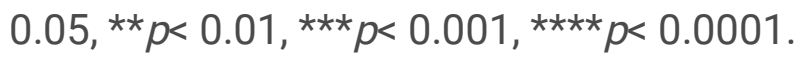

e, Representative images showing the condition of glutamatergic neurons in Day-3 hApoE3 ${ }^{\text {Glu }}$ (left), hA $\beta_{1}$. ${ }_{42}$ Glu (up-right), and $\mathrm{hA} \beta_{1-42}$ Glu nematodes under different conditions.

f. PEP protected against $A \beta$-induced glutamatergic neuronal degeneration in Day-3 hA $\beta_{1-42}$ Glu nematodes. Five glutamatergic neurons including LUA(R), LUA(L), PLM(R), PLM $(L)$, and PVR were used for data analysis. Data were from three biological repeats. One-way ANOVA followed by Tukey's multiple comparisons test was used for data analysis with ns, no significance; ${ }^{\star} p<0.05,{ }^{\star \star} p<0.01,{ }^{\star \star \star} p<0.001$, $\star \star \star \star x<0.0001$.

g-i, PEP attenuated high glutamate $(5 \mathrm{mM})$-induced cell death in HT-22 cells under different conditions. Varied concentrations $(12.5-100 \mu \mathrm{g} / \mathrm{mL})$ of PEP were used in the experiments. Data were from three biological repeats. One-way ANOVA followed by Tukey's multiple comparisons test was used for data analysis with ns, no significance; ${ }^{*} p<0.05,{ }^{* \star} p<0.01,{ }^{* \star *} p<0.001,{ }^{* \star \star \star} p<0.0001$. 
a

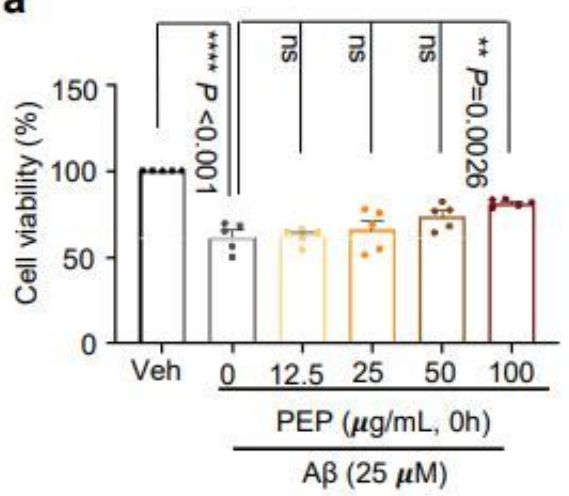

d
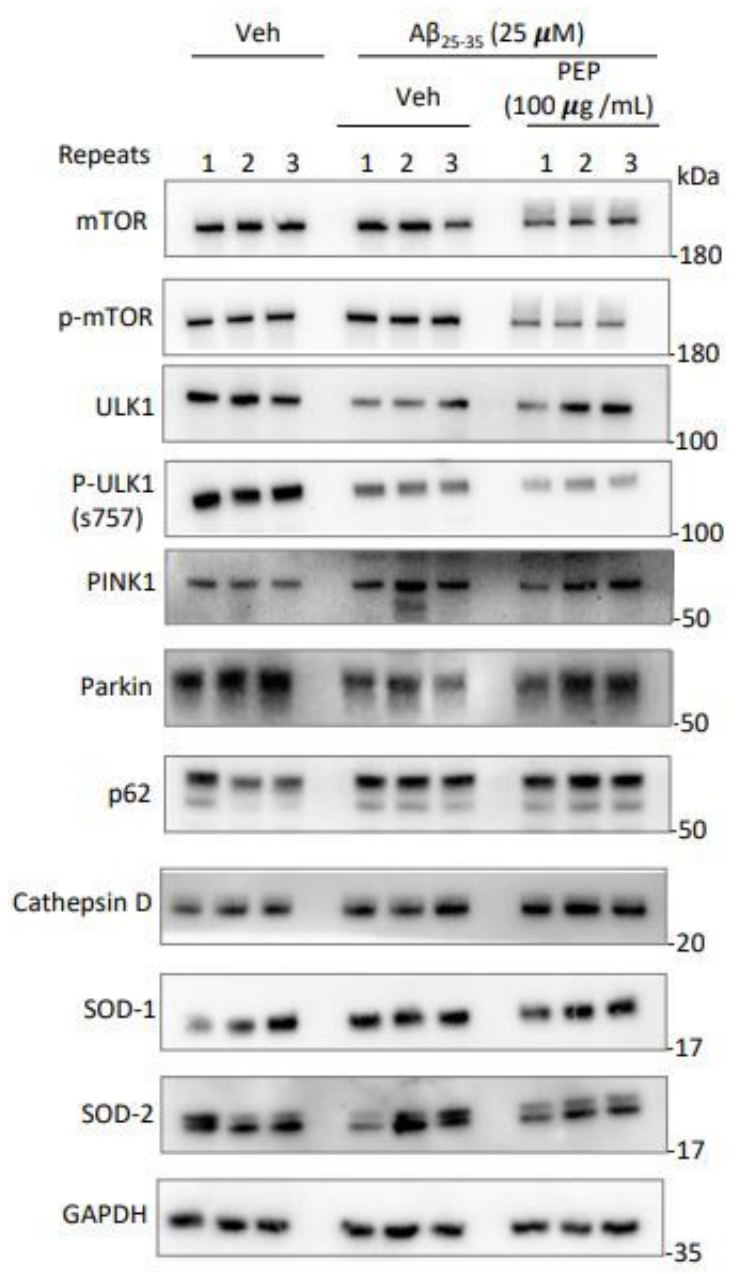

b

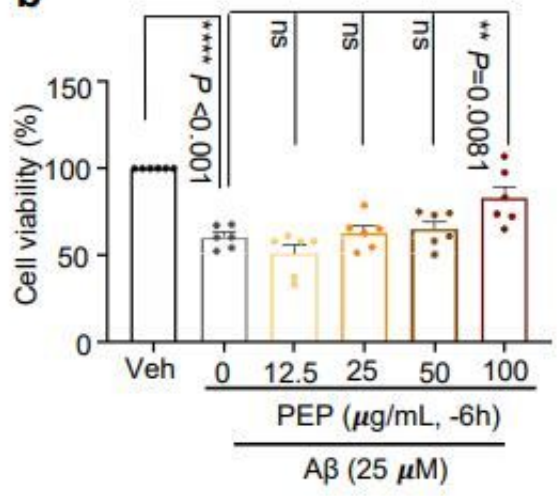

e

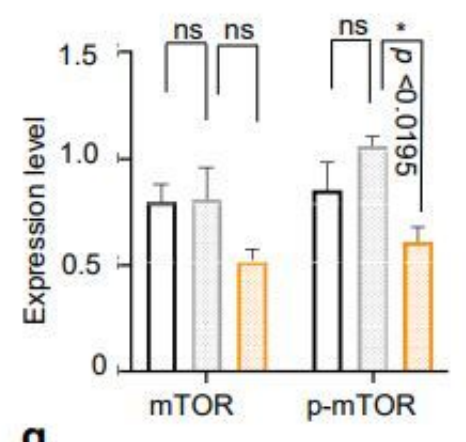

g

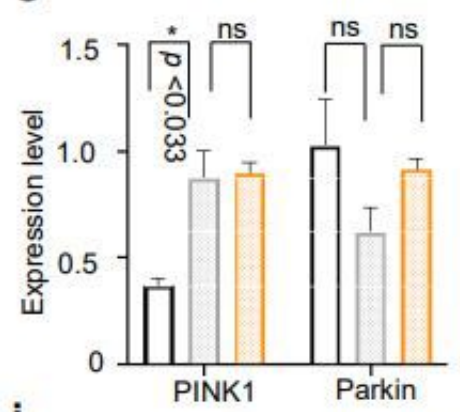

i

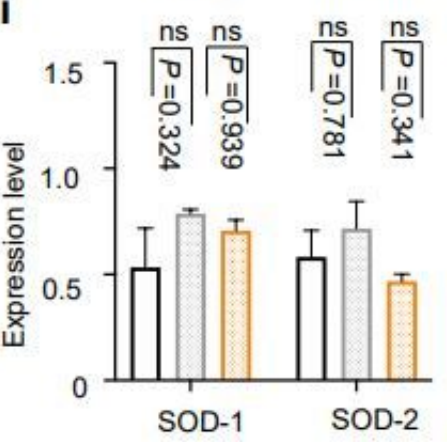

C

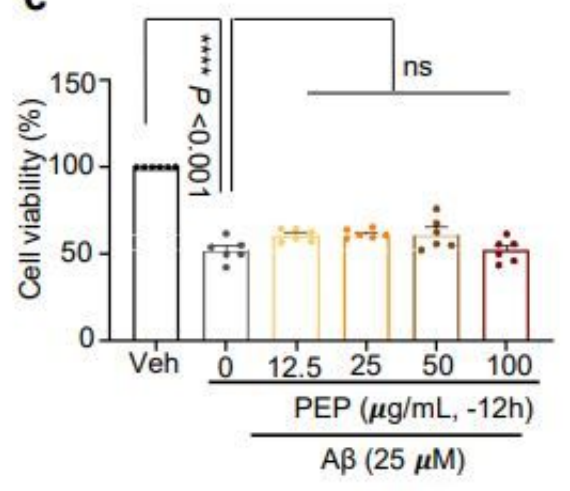

f

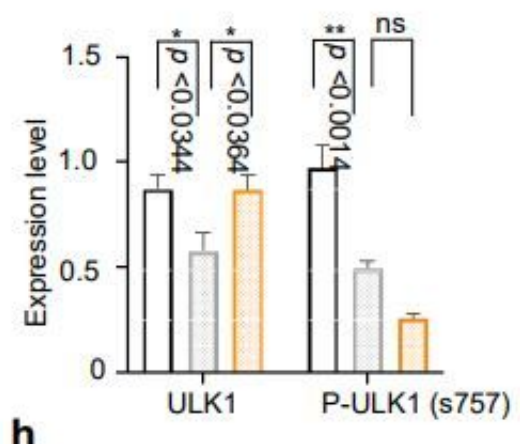

h

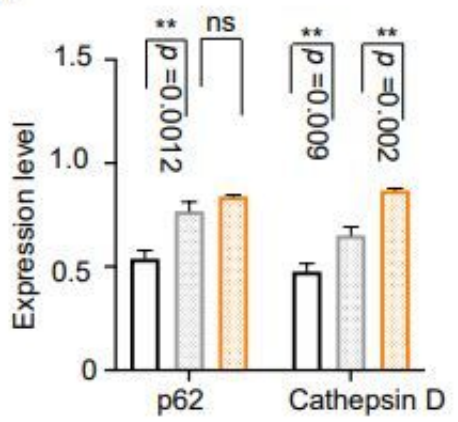

$\square$ Veh

$A \beta_{25-35}(25 \mu \mathrm{M})$

$\square A \beta_{25.35}(25 \mu \mathrm{M})+\operatorname{PEP}(100 \mu \mathrm{g} / \mathrm{mL})$

\section{Figure 3}

PEP protects against $A \beta_{25-35}$-induced neurotoxicity in human SH-SY5Y-differentiated neuronal-like cells.

a-c, PEP attenuated $A \beta_{25-35}$-induced cell death in SHSY5Y-differentiated neuronal-like cells under designated conditions. Varied concentration (12.5 - $100 \mu \mathrm{g} / \mathrm{mL})$ of PEP were used in the experiments. 
Data were from three biological repeats. One-way ANOVA followed by Tukey's multiple comparisons test was used for data analysis with ns, no significance; ${ }^{\star} p<0.05,{ }^{\star} * p<0.01,{ }^{\star} * \star p<0.001,{ }^{\star \star \star \star} p<0.0001$.

d, Effects of $A \beta_{25-35}(25 \mu \mathrm{M})$ and PEP $(100 \mu \mathrm{g} / \mathrm{mL})$ on the expression of designated proteins in SH-SY5Ydifferentiated neuronal-like cells.

e-h, Quantifications of the expression level of designated proteins as compared to GAPDH. Data were from three biological repeats. One-way ANOVA followed by Tukey's multiple comparisons test was used for data analysis with ns, no significance; ${ }^{*} p<0.05$, ${ }^{*} p<0.01$, ${ }^{\star \star *} p<0.001$, ${ }^{* \star \star *} p<0.0001$.

a

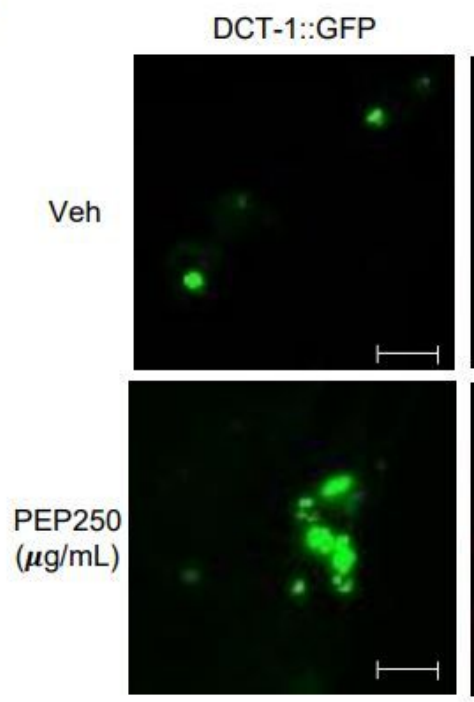

C

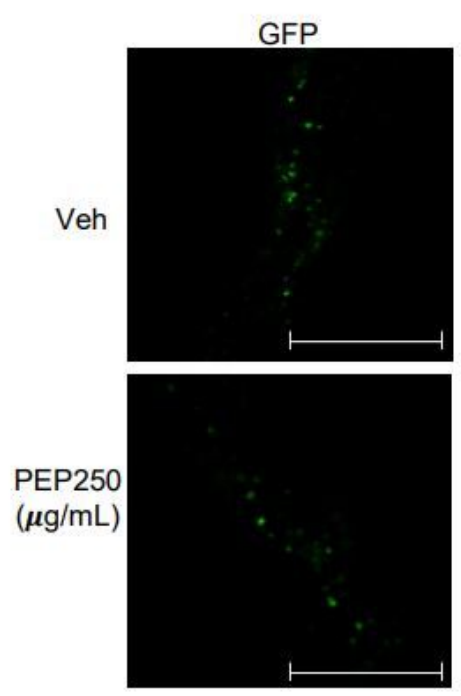

LGG-1::DsRed
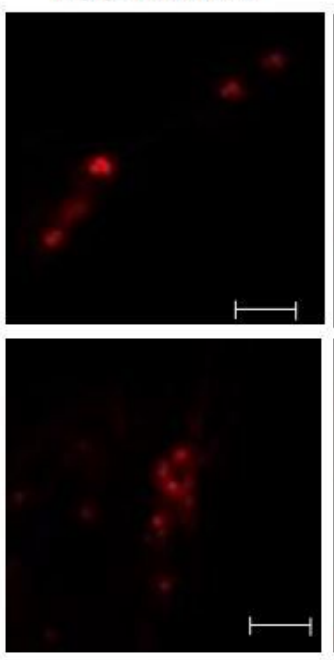

DsRed
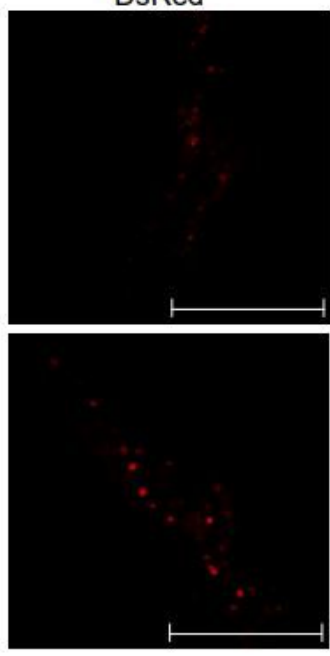

Merge

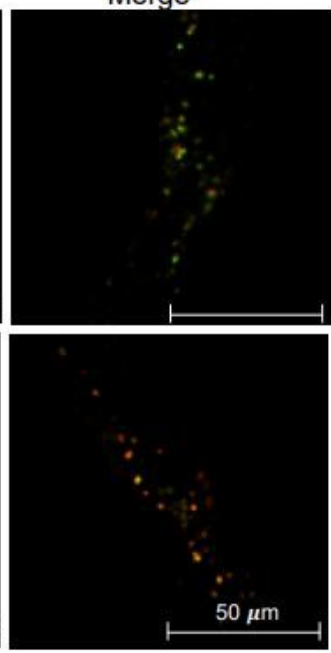

b
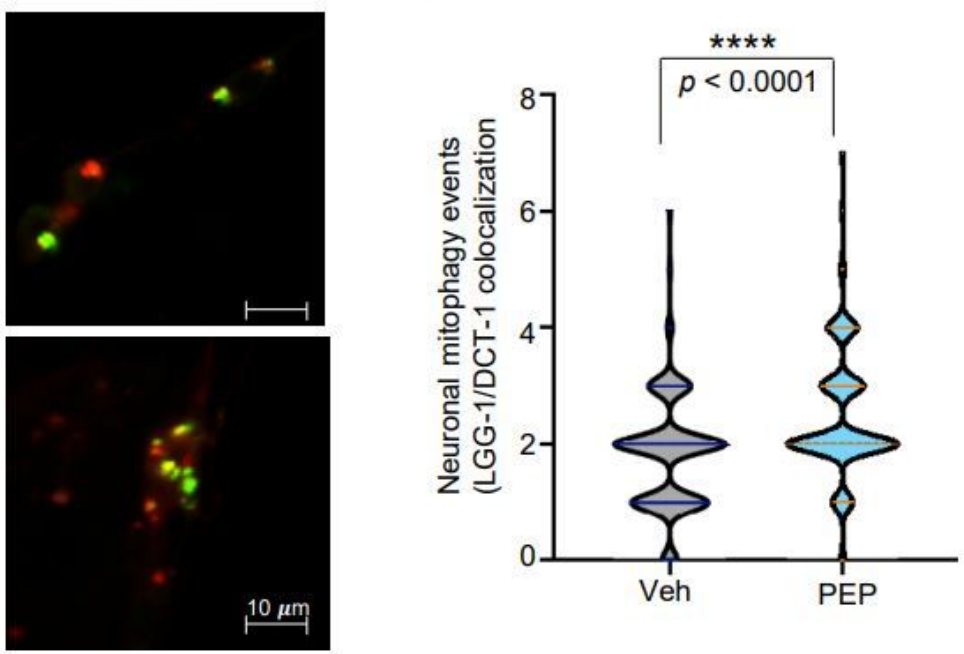

d

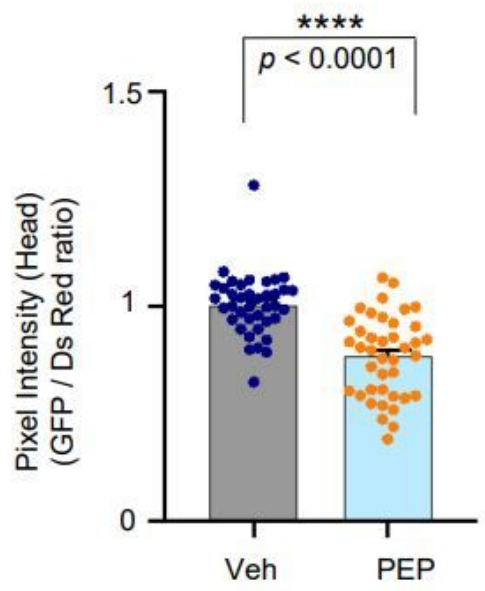

Figure 4

PEP induces mitophagy in $C$. elegans neurons. 
a, Representative images showing the LGG-1 and DCT-1 coloclization in control or PEP $(250 \boldsymbol{\mu} \mathrm{g} / \mathrm{mL})$ extract fed adult Day-1 nematodes. Scale bar, $10 \mu \mathrm{m}$.

b, PEP enhanced LGG-1 and DCT-1 co-localization which indicate mitophagy events. Data were from two biological repeats with a total of 38 to 45 nematodes used for data quantification. One-way ANOVA followed by Tukey's multiple comparisons test was used for data analysis with ns, no significance; ${ }^{\star} p<$

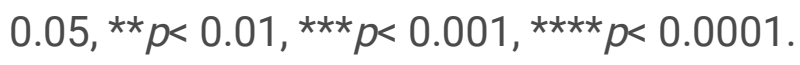

c, Representative images showing the GFP/DsRed ratio in control or PEP (250 $\boldsymbol{\mu g} / \mathrm{mL})$ extract fed adult Day-1 mtRosella nematodes. Scale bar, $50 \mu \mathrm{m}$.

d, PEP reduced GFP/DsRed ratio indicating increased mitophagy. Data were from three biological repeats with 40 nematodes. One-way ANOVA followed by Tukey's multiple comparisons test was used for data

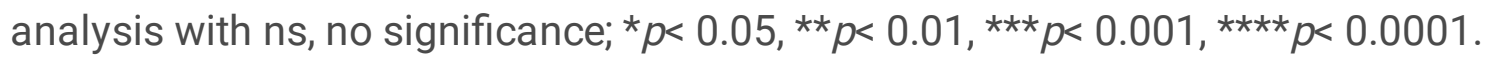


a

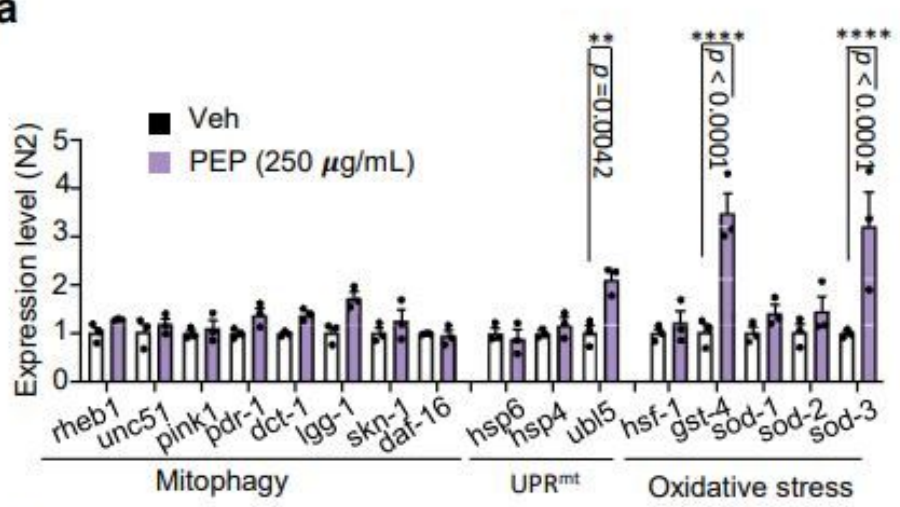

C

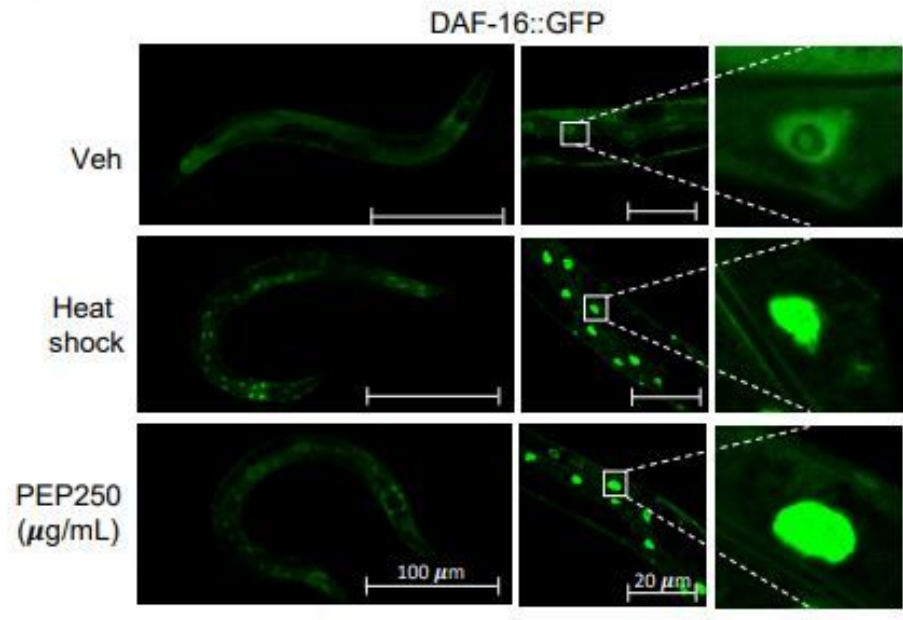

f

- Veh (Naĩve) •PEP $250 \mu \mathrm{g} / \mathrm{mL}$ (Naĩve)

-Veh (IA) - PEP $250 \mu \mathrm{g} / \mathrm{mL}$ (IA)

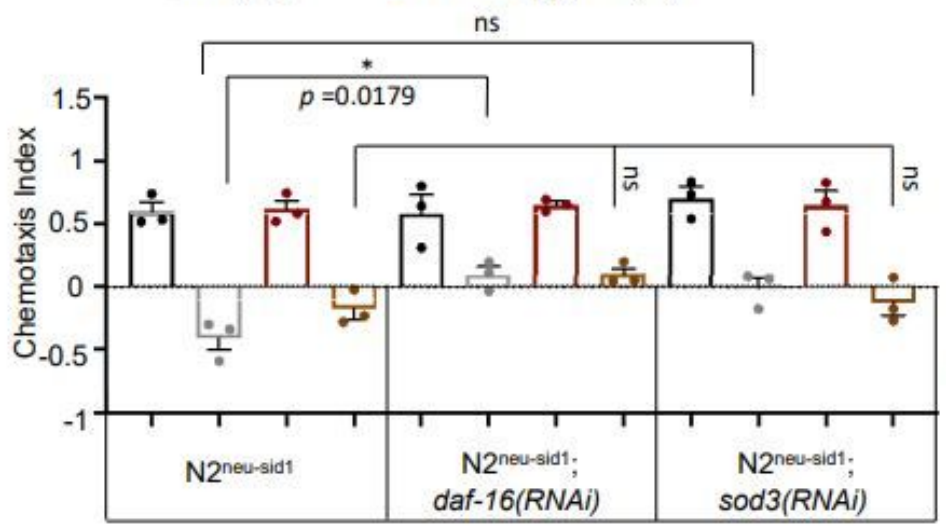

b

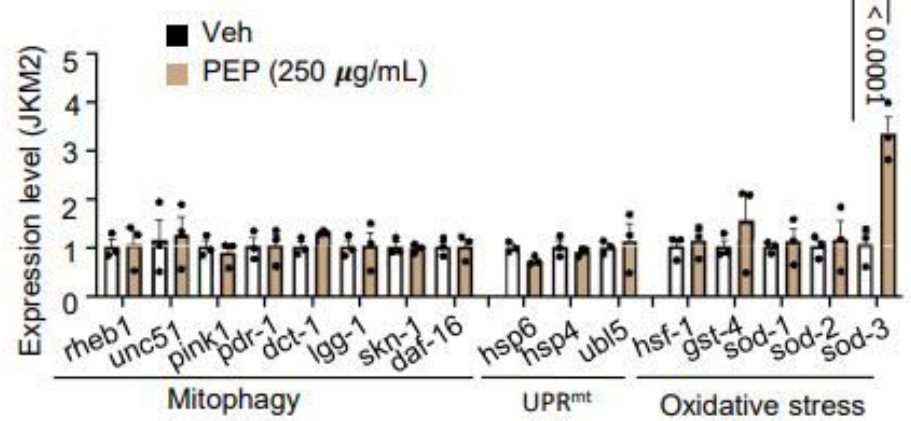

d

e
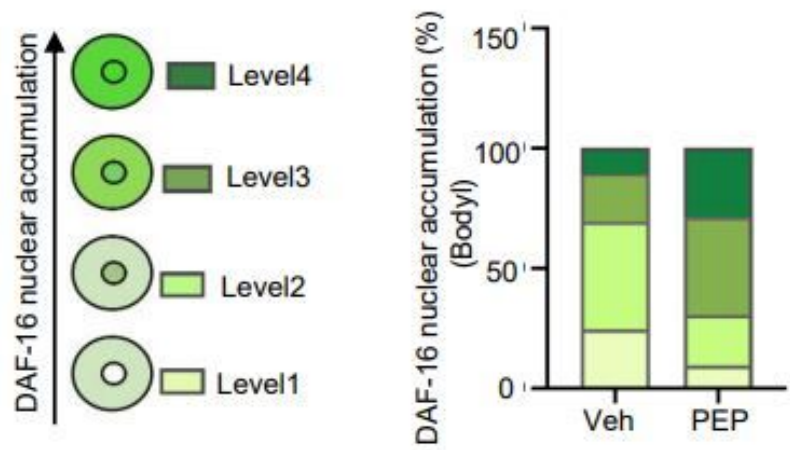

g

- Veh (Naĩve) - PEP $250 \mu \mathrm{g} / \mathrm{mL}$ (Naĩve)

- Veh (IA) - PEP $250 \mu \mathrm{g} / \mathrm{mL}$ (IA)

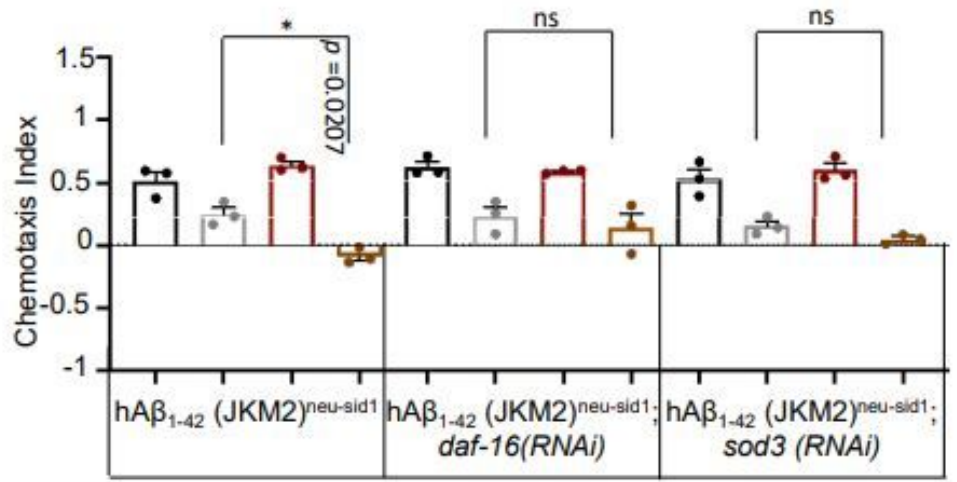

Figure 5

PEP induces DAF-16 nuclear localization, leading to higher transcriptional regulation of downstream genes.

a-b, Effect of PEP on mitochondria-related gene expression in Day-1 WT (N2) and hA $\beta_{1-42}$ (JKM2) nematodes. Data were from three biological repeats (each biological repeat includes three technical 
repeats). Two-way ANOVA followed by Sidak's multiple comparisons test was used for data analysis with

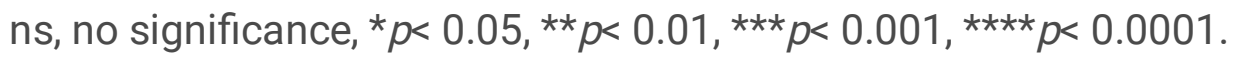

C, Images showing differential subcellular distribution of DAF-16 in vehicle (left), Heat shock $\left(37^{\circ} \mathrm{C}\right.$, $30 \mathrm{~min}$, positive control) (middle), and PEP (250 $\boldsymbol{\mu g} / \mathrm{mL})$ nematodes(right). Scale bars, $100 \boldsymbol{\mu m}$ and 20 $\boldsymbol{\mu} \mathrm{m}$, respectively.

d. PEP promoted DAF-16 nuclear translocation in adult Day-1 nematodes. Nematodes were treated with PEP $(250 \mu \mathrm{g} / \mathrm{mL})$ from L4 stage. Data were from two biological repeats with 40 nematodes.

ef, Knocked down of neuronal daf-16 or sod-3 gene affected PEP-induced memory improvement in the $\mathrm{N} 2{ }^{\text {neu-sid1 }}$ (e) and hA $\beta_{1-42}(J K M 2)^{\text {neu-sid1 }}$ (f) nematodes. Data were from four biological repeats with the results shown in mean \pm S.E.M. One-way ANOVA followed by Tukey's multiple comparisons test was used for data analysis with ns, no significance, ${ }^{*} p<0.05,{ }^{*} p<0.01, * \star * p<0.001, * \star \star * x<0.0001$.

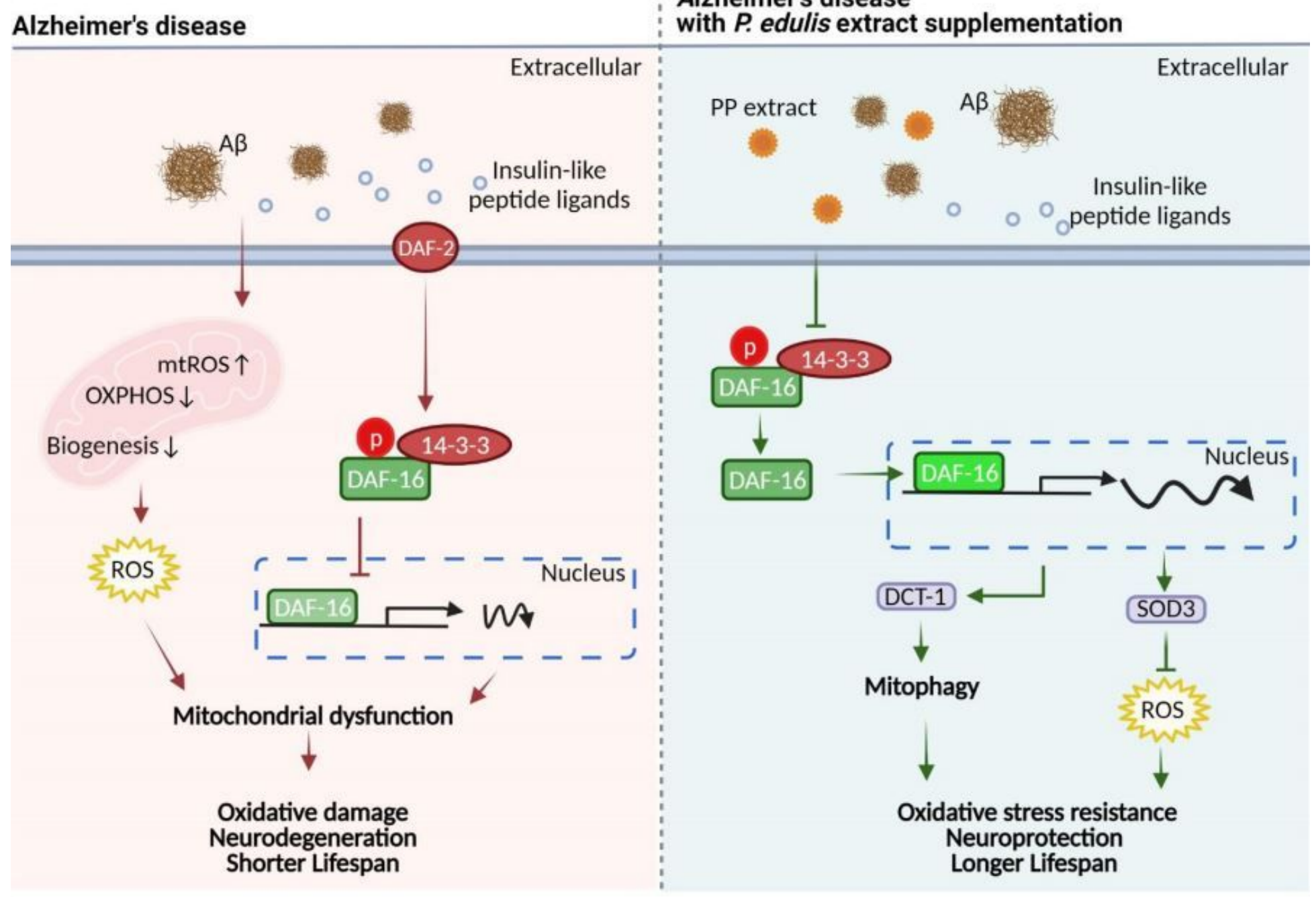

Alzheimer's disease

with $P$. edulis extract supplementation 
Figure 6

Schematic representation showing proposed anti-AD mechanism by the pericarp extract of $P$. edulis (Created with BioRender.com). The pericarp extract protects against $A \beta$ - and pTau-induced neurodegeneration and memory loss in nematode models of AD via induction of DAF-16/FOXO3dependent mitophagic and anti-oxidative pathways.

\section{Supplementary Files}

This is a list of supplementary files associated with this preprint. Click to download.

- Sfig1.jpg

- Sfig2.jpg

- Supplementarydata.docx 